\title{
The structure of extremals of a class of second order variational problems
}

by

\author{
Moshe MARCUS and Alexander J. ZASLAVSKI \\ Department of Mathematics, \\ Technion-Israel Institute of Technology, Haifa, 32000, Israel
}

\begin{abstract}
We study the structure of extremals of a class of second order variational problems without convexity, on intervals in $R_{+}$. The problems are related to a model in thermodynamics introduced in [7]. We are interested in properties of the extremals which are independent of the length of the interval, for all sufficiently large intervals. As in $[12,13]$ the study of these properties is based on the relation between the variational problem on bounded, large intervals and a limiting problem on $R_{+}$. Our investigation employs techniques developed in $[10,12,13]$ along with turnpike techniques developed in $[16,17]$.
\end{abstract}

(C) 1999 L'Association Publications de l'Institut Henri Poincaré. Published by Elsevier B.V. All rights reserved

Key words: Turnpike properties, $(f)$-good functions, periodic minimizers.

RÉsumÉ. - On étudie la structure des extrêmales d'une classe de problèmes variationnels non convexes du deuxième ordre, sur des intervalles de $R_{+}$. Ces problèmes sont reliés à un modèle thérmodynamique introduit dans [7]. Nous nous intéressons aux propriétés des extrêmales qui ne dependent pas de la longeur des intervalles, pourvu que ceux-ci soient assez grands. Comme dans $[12,13]$ l'etude de ces propriétés s'appuie sur la relation entre le problème variationnel sur de grands intervalles bornés et un problème limite sur $R_{+}$. Notre travail emploie des techniques développées dans $[10,12,13]$ ainsi que dans $[16,17]$.

(C) 1999 L'Association Publications de l'Institut Henri Poincaré. Published by Elsevier B.V. All rights reserved

1991 Mathematics Subject Classification: 49 J 99; 58 F 99.

Annales de l'Institut Henri Poincaré - Analyse non linéaire - 0294-1449

Vol. 16/99/05/

(C) 1999 L'Association Publications de l'Institut Henri Poincaré. Published by Elsevier B.V. All rights reserved 


\section{INTRODUCTION}

In this paper we investigate the structure of optimal solutions of variational problems associated with the functional

$$
J^{f}(D ; w)=|D|^{-1} \int_{D} f\left(w(t), w^{\prime}(t), w^{\prime \prime}(t)\right) d t, \quad \forall w \in W^{2,1}(D),
$$

where $D$ is a bounded interval on the real line and $f \in C\left(R^{3}\right)$ belongs to a space of functions to be described below. Specifically we shall consider the problems,

$$
\inf \left\{J^{f}(D ; w): w \in W^{2,1}(D)\right\}
$$

and, for $D=\left(T_{1}, T_{2}\right)$,

$\left(P_{D}^{x, y}\right)$

$$
\inf \left\{J^{f}(D ; w): w \in W^{2,1}(D),\left(w, w^{\prime}\right)\left(T_{1}\right)=x,\left(w, w^{\prime}\right)\left(T_{2}\right)=y\right\} .
$$

In connection with these we shall also study the following problem on the half line:

$$
\left(P_{\infty}\right) \quad \inf \left\{J^{f}(w): w \in W_{l o c}^{2,1}(0, \infty)\right\},
$$

where

$$
J^{f}(w)=\liminf _{T \rightarrow \infty} J^{f}((0, T) ; w) .
$$

This can be seen as a limiting problem for $\left(P_{D}\right)$ as $|D| \rightarrow \infty$. Variational problems of this type were considered by Leizarowitz and Mizel [10]. Similar constrained problems (involving a mass constraint), were studied by Coleman, Marcus and Mizel [7] and by Marcus [12,13]. The constrained problems were conceived as models for determining the thermodynamical equilibrium states of unidimensional bodies involving 'second order' materials (see [7]).

Let $G=G(p, r)$ be a function in $C^{4}\left(R^{2}\right)$ such that

$$
\begin{aligned}
& \partial^{2} G / \partial r^{2}(p, r)>0, \\
& G(p, r) \geq|r|^{\gamma}-b_{1}|p|^{\beta}-b_{0}, \quad \forall(p, r) \in R^{2},
\end{aligned}
$$

where $b_{1}, b_{0}$ are positive constants, $1 \leq \beta \leq \gamma$ and $\gamma>1$. In addition assume that,

$$
\max \{|G(p, r)|,|\partial G / \partial r(p, r)|,|\partial G / \partial p(p, r)|\} \leq M(|p|)\left(1+|r|^{\gamma}\right),
$$

where $M:[0, \infty) \rightarrow[0, \infty)$ is a continuous function. A typical example is $G(p, r)=r^{2}-b p^{2}$. 
Let $\alpha, b_{2}, b_{3}$ be positive numbers, with $\alpha>\beta$, and let

$$
\mathfrak{L}=\mathfrak{L}\left(\alpha, b_{2}, b_{3}\right)=\left\{\phi \in C^{2}\left(R^{1}\right): \phi(t) \geq b_{3}|t|^{\alpha}-b_{2}, \quad \forall t \in R^{1}\right\}
$$

The space $\mathfrak{L}$ will be equipped with the standard topology of $C^{2}$. Finally denote,

$$
\mathfrak{L}_{G}=\mathfrak{L}_{G}\left(\alpha, b_{2}, b_{3}\right)=\left\{F_{\phi}: \phi \in \mathfrak{L}\left(\alpha, b_{2}, b_{3}\right)\right\},
$$

where,

$$
F_{\phi}(w, p, r)=\phi(w)+G(p, r), \quad \forall(w, p, r) \in R^{3} .
$$

The relation between the minimizers of $\left(P_{D}\right)$ (for large $|D|$ ) and those of $\left(P_{\infty}\right)$ plays a crucial role in our study of their structure. This relation was first investigated by Marcus $[12,13]$ where it was used in order to derive structural properties of minimizers of problem $\left(P_{D}\right)$ and of related constrained problems, in the case $f=r^{2}-b p^{2}+\phi(w)$. In the present paper we pursue this investigation combining techniques of $[12,13]$ with turnpike techniques as in Zaslavski [16, 17].

One of our main results is the uniqueness of periodic minimizers of $\left(P_{\infty}\right)$ which is generically valid in a very precise sense.

For every potential $\phi \in \mathfrak{L}\left(\alpha, b_{2}, b_{3}\right)$ there exists a family of arbitrarily small perturbations $\left\{\phi_{s}=\phi+s \theta: 0<s<1\right\}$, such that problem $\left(P_{\infty}\right)$ with $f=F_{\phi_{s}}$ possesses a unique (up to translation) periodic minimizer.

The function $\theta$ can be explicitly constructed in terms of the extremal values of periodic minimizers of $\left(P_{\infty}\right)$ with $f=F_{\phi}$. Combining this result with a recent result of Zaslavski [18], we show that for each potential $\phi_{s}$ in this family, the corresponding integrand $F_{\phi_{s}}$ possesses an asymptotic turnpike property, which involves the behaviour of the limit set of minimizers of $\left(P_{\infty}\right)$. Finally, we show that this asymptotic property can be used in order to derive detailed information on the structure of minimizers of problem $\left(P_{D}\right)$ for all sufficiently large intervals $D$. In this last part the results are valid not only in the generic sense, but apply to every $f \in \mathfrak{L}_{G}$.

A brief comparison of the present results with those of [13]: In the present work, as in [13], the structure of minimizers of $\left(P_{D}\right)$ is described by observing their behaviour in a 'window' of fixed length (independent of $|D|)$ which can be placed anywhere in $D$. The results of [13] apply to every integrand of the form $f=r^{2}-b p^{2}+\phi(w)$, for a class of potentials $\phi$ which 
includes the standard two-well potentials. The behaviour of minimizers of $\left(P_{D}\right)$ in a 'window' is described by integral estimates, involving 'mass' and 'energy'. The present results are in part generic, but they deal with a very large class of integrands and the behaviour of minimizers in a 'window' is described by pointwise estimates which provide considerably more detailed information.

For a precise statement of the results mentioned above we need some additional notation and definitions.

Let $\mu(f)$ denote the infimum in $\left(P_{\infty}\right)$ with $f \in \mathfrak{L}_{G}$. Leizarowitz and Mizel [10] proved that, if $\mu(f)<\inf _{(w, s) \in R^{2}} f(w, 0, s)$, then $\left(P_{\infty}\right)$ possesses a periodic minimizer. Zaslavski [15] showed that the result remains valid for all $f \in \mathfrak{L}_{G}$.

For $w \in W_{l o c}^{2,1}(0, \infty)$ put,

$$
\eta^{f}(T, w)=\left(J^{f}((0, T) ; w)-\mu(f)\right) T, T \in(0, \infty) .
$$

Then, either $\sup _{0<T<\infty}\left|\eta^{f}(T, w)\right|<\infty$ or $\lim _{T \rightarrow \infty} \eta^{f}(T, w)=+\infty$. Furthermore, if $\eta^{f}(\cdot, w)$ is bounded then $w$ and $w^{\prime}$ are bounded [15, Prop. 3.1].

Let $w$ be an $(f)$-minimizer of $\left(P_{\infty}\right)$. We shall say that $w$ is $(f)$-good if $\eta^{f}(\cdot, w)$ is bounded. Equivalently, $w$ is $(f)$-good if and only if there exists a constant $c(w)$ such that,

$$
\left|J^{f}(D ; w)-\mu(f)\right| \leq c(w) /|D|
$$

for every bounded interval $D$.

We shall say that $w$ is optimal on compacts, or briefly c-optimal, if $w \in W_{l o c}^{2,1}(0, \infty) \cap W^{1, \infty}(0, \infty)$ and, for every bounded interval $D$, the restriction $\left.w\right|_{D}$ is a minimizer of $\left(P_{D}^{x, y}\right)$, where $x, y$ are the values of $\left(w, w^{\prime}\right)$ at the end points of the interval. By a result of Marcus [13, Th. 4.2(vi)], if the integrand $f$ is of the form $f(w, p, r)=r^{2}-b p^{2}+\phi(w)$, then every c-optimal minimizer of $\left(P_{\infty}\right)$ is $(f)$-good. In fact the result remains valid for the more general class of integrands studied here, (see Proposition 2.3 below).

For $w \in W_{\text {loc }}^{2,1}(0, \infty) \cap W^{1, \infty}(0, \infty)$ let $\Omega(w)$ denote the set of limiting points of $\left(w, w^{\prime}\right)$ as $t \rightarrow \infty$.

Definimon 1.1. - Let $f \in \mathfrak{L}_{G}$. We say that $\int$ has the asymptotic turnpike property, or briefly (ATP), if there exists a compact set $H(f) \subset R^{2}$ such that $\Omega(w)=H(f)$ for every $(f)$-good minimizer $w$.

Clearly, if $f$ has (ATP) and $v$ is a periodic $(f)$-minimizer of $\left(P_{\infty}\right)$ then, $H(f)=\left\{\left(v, v^{\prime}\right)(t): 0 \leq t<\infty\right\}$. 
The asymptotic turnpike property for optimal control problems was studied in $[4,5]$. The more standard turnpike property (for problems on finite intervals) is well known in mathematical economics and several variants of it have been studied (see, e.g. [11] and [6, Ch.4 and 6]). Here we shall consider, besides (ATP), the strong turnpike property, or briefly (STP), which is defined as follows.

Definition 1.2. - Let $f \in \mathfrak{L}_{G}$ and let $w$ be a periodic $(f)$-minimizer of $\left(P_{\infty}\right)$ with period $T_{w}>0$. We say that $f$ has the strong turnpike property if, for every $\epsilon>0$ and every bounded set $K \subset R^{2}$, there exists $L>0$ such that every minimizer $v$ of $\left(P_{(0, T)}^{x, y}\right)$, with $x, y \in K$ and $T>T_{w}+2 L$, satisfies the following:

For every $a \in\left[L, T-L-T_{w}\right]$ there exists $\bar{a} \in\left[0, T_{w}\right)$ such that,

$$
\left|\left(v, v^{\prime}\right)(a+t)-\left(w, w^{\prime}\right)(\vec{a}+t)\right| \leq \epsilon, \quad \forall t \in\left[0, T_{w}\right] .
$$

Note that (STP) implies uniqueness up to translation for periodic minimizers of $\left(P_{\infty}\right)$. Furthermore, if $f$ has (STP), the structural information contained in (1.8) extends to arbitrary minimizers of the unconstrained problem $\left(P_{(0, T)}\right)$. More precisely we have,

Proposition 1.1. - Suppose that $f \in \mathfrak{L}_{G}$ possesses (STP). Let $w$ be the (unique) periodic minimizer of $\left(P_{\infty}\right)$ whose period will be denoted by $T_{w}$. Then, given $\epsilon>0$, there exists $L>0$ such that every minimizer $v$ of $\left(P_{(0, T)}\right)$ with $T>T_{w}+2 L$ satisfies (1.8) for every $a \in\left[L, T-L-T_{w}\right]$ and some $\bar{a} \in\left[0, T_{w}\right)$ depending on $v$ and $a$.

This is a consequence of the fact that the set of minimizers of $\left(P_{(0, T)}\right)$ is bounded in $C^{1}[0, T]$ by a constant $A$ independent of $T$, (see [12, Lemma 2.2]).

Our main results are the following.

THEOREM 1.1. For $f \in \mathfrak{L}_{G}$, (STP) holds if and only if (ATP) holds.

THEOREM 1.2. - For every $\phi \in \mathfrak{L}$ there exists a non-negative function $\theta \in C^{\infty}\left(R^{1}\right)$ with $\theta^{(m)} \in L^{\infty}\left(R^{1}\right), m=0,1, \ldots$, such that for every $s \in(0,1)$, problem $\left(P_{\infty}\right)$ with $f=F_{\phi+s \theta}$ possesses a unique (up to translation) periodic minimizer.

THEOREM 1.3. - (i) For every $\phi \in \mathfrak{L}$ there exists a function $\theta$ as in Theorem 1.2 such that,

$$
F_{\phi+s \theta} \text { possesses }(A T P), \forall s \in(0,1) \text {. }
$$

Vol. $16, n^{\circ}$ 5-1999. 
(ii) (ATP) holds generically in $\mathfrak{L}_{G}$, in the following sense as well: there exists a countable intersection of open everywhere dense sets in $\mathfrak{L}$, say $\mathfrak{F}_{G}$, such that

$$
\phi \in \mathfrak{F}_{G} \Longrightarrow F_{\phi} \text { possesses (ATP). }
$$

A result related to the second part of Theorem 1.3 was obtained by Zaslavski [16], who established the generic validity of (ATP) in a larger space, in a weaker sense.

The proofs of these theorems, in a slightly more general form, are presented in sections 2 (Theorem 1.1) and 3 (Theorems 1.2, 1.3). In addition, in section 3 , we establish a number of properties of periodic minimizers of $\left(P_{\infty}\right)$ which apply to every $f \in \mathfrak{L}_{G}$ and may be of independent interest.

\section{EQUIVALENCE OF (ATP) AND (STP)}

In this section we shall establish Theorem 1.1 for problems involving a larger family of integrands $f$. Put,

$$
\mathfrak{A}=\left\{f \in C\left(R^{3}\right):\left|f\left(x_{1}, x_{2}, x_{3}\right)\right| \rightarrow \infty \text { as }\left|x_{3}\right| \rightarrow \infty,\right.
$$

uniformly with respect to $\left(x_{1}, x_{2}\right)$ in compact sets\}.

$\mathfrak{A}$ will be equiped with the uniformity determined by the base,

$$
\begin{aligned}
E(N, \epsilon)=\{ & (f, g) \in \mathfrak{A} \times \mathfrak{A}: \\
& |f(x)-g(x)| \leq \epsilon, \quad\left(x=\left(x_{1}, x_{2}, x_{3}\right) \in R^{3},\right. \\
& \left.\left|x_{i}\right| \leq N, i=1,2,3\right), \\
& 1-\epsilon \leq(|f(x)|+1) /(|g(x)|+1) \leq 1+\epsilon, \\
& \left.\left(x \in R^{3},\left|x_{1}\right|,\left|x_{2}\right| \leq N\right)\right\}
\end{aligned}
$$

where $N$ and $\epsilon$ are positive numbers. It is easy to verify that the uniform space $\mathfrak{A}$ is metrizable and complete [8].

Let $a=\left(a_{1}, a_{2}, a_{3}, a_{4}\right) \in R^{4}, a_{i}>0, i=1,2,3,4$ and let $\alpha, \beta, \gamma$ be real numbers such that $1 \leq \beta<\alpha, \beta \leq \gamma$ and $\gamma>1$. Denote by $\mathfrak{M}=\mathfrak{M}(\alpha, \beta, \gamma, a)$ the family of functions $\{f\}$ such that

(i) $f \in \mathfrak{A} \cap C^{2}\left(R^{3}\right), \partial f / \partial x_{2} \in C^{2}\left(R^{3}\right), \partial f / \partial x_{3} \in C^{3}\left(R^{3}\right)$,

(ii) $\partial^{2} f / \partial x_{3}^{2}>0$,

(iii) $f(x) \geq a_{1}\left|x_{1}\right|^{\alpha}-a_{2}\left|x_{2}\right|^{\beta}+a_{3}\left|x_{3}\right|^{\gamma}-a_{4}$,

(iv) $(|f|+|\nabla f|)(x) \leq M_{f}\left(\left|x_{1}\right|+\left|x_{2}\right|\right)\left(1+\left|x_{3}\right|^{\gamma}\right), \quad \forall x \in R^{3}$, 
where $M_{f}:[0, \infty) \mapsto[0, \infty)$ is a continuous function depending on $f$. Finally, let $\overline{\mathfrak{M}}$ denote the closure of $\mathfrak{M}$ in $\mathfrak{A}$. The notations and definitions presented in the introduction with respect to $f \in \mathfrak{L}_{G}$ apply equally well to $f \in \mathfrak{M}$ and the various statements quoted there remain valid in this context. Put,

$$
I^{f}\left(T_{1}, T_{2}, w\right)=\int_{T_{1}}^{T_{2}} f\left(w(t), w^{\prime}(t), w^{\prime \prime}(t)\right) d t
$$

where $-\infty<T_{1}<T_{2}<+\infty, w \in W^{2,1}\left(T_{1}, T_{2}\right)$ and $f \in \overline{\mathfrak{M}}$.

For $T>0, x, y \in R^{2}, f \in \overline{\mathfrak{M}}$, put

$$
\begin{aligned}
U_{T}^{f}(x, y):=\inf \left\{I^{f}(0, T, w)\right. & : w \in W^{2,1}(0, T), \\
\left(w, w^{\prime}\right)(0) & \left.=x,\left(w, w^{\prime}\right)(T)=y\right\} .
\end{aligned}
$$

Let $v \in W^{2,1}(D)$ where $D=\left(T_{1}, T_{2}\right)$ is a bounded interval. Given $\delta>0$, we shall say that $v$ is an $(f, \delta)$-approximate minimizer in $D$ if,

$$
\begin{gathered}
I^{f}\left(T_{1}, T_{2}, v\right) \leq U_{|D|}^{f}\left(X_{v}\left(T_{1}\right), X_{v}\left(T_{2}\right)\right)+\delta, \\
X_{v}(t)=\left(v(t), v^{\prime}(t)\right), \quad t \in D .
\end{gathered}
$$

For $x \in R^{n}, B \subset R^{n}$ put $d(x, B):=-\inf \{|x-y|: y \in B\}$ (where $|\cdot|$ is the Euclidean norm) and denote by $\operatorname{dist}(A, B)$ the distance in the Hausdorff metric between two subsets $A, B$ of $R^{n}$.

We claim that:

LEMMA 2.1. - Suppose that $f \in \overline{\mathfrak{M}}$ and that $v$ is an $(f)$-good function. Then, given $\delta>0$ there exists $T_{\delta}>0$ such that, for every bounded interval $\left(T, T^{\prime}\right)$ with $T \geq T_{\delta}$,

$$
I^{f}\left(T, T^{\prime}, v\right) \leq U_{T^{\prime}-T}^{f}\left(X_{v}(T), X_{v}\left(T^{\prime}\right)\right)+\delta,
$$

i.e. $v$ is an $(f, \delta)$-approximate minimizer in $\left(T, T^{\prime}\right)$.

Proof. - If the claim is not valid there exists a sequence of disjoint intervals $D_{n}=\left(T_{n}, T_{n}^{\prime}\right), n=1,2, \ldots$ with $T_{n} \rightarrow \infty$ such that,

$$
I^{f}\left(T_{n}, T_{n}^{\prime}, v\right)-U_{T_{n}^{\prime}-T_{n}}^{f}\left(x_{n}, y_{n}\right) \geq \delta, \quad n=1,2, \ldots,
$$

where $x_{n}=X_{v}\left(T_{n}\right)$ and $y_{n}=X_{v}\left(T_{n}^{\prime}\right)$. Let $h_{n}$ denote a minimizer of problem $\left(P_{D_{n}}^{x_{n}, y_{n}}\right)$ and let $\tilde{v}$ be the function on $[0, \infty)$ defined as follows,

$$
\tilde{v}(t)=v(t), t \in[0, \infty) \backslash \cup_{n} D_{n}, \quad \tilde{v}(t)=h_{n}(t), t \in D_{n}, n=1,2, \ldots
$$


Then $\tilde{v} \in W_{l o c}^{2,1}(0, \infty)$ and

$$
\eta^{f}(T, \tilde{v})=\left(I^{f}(0, T, \tilde{v})-I^{f}(0, T, v)\right)+\eta^{f}(T, v) .
$$

Since $\eta^{f}(\cdot, v)$ is bounded, say by $M$, it follows that,

$$
\eta^{f}\left(T_{n}^{\prime}, \tilde{v}\right) \leq M-\sum_{k=1}^{n}\left(I^{f}\left(T_{k}, T_{k}^{\prime}, v\right)-U_{T_{k}^{\prime}-T_{k}}^{f}\left(x_{k}, y_{k}\right)\right)
$$

This inequality and (2.5) imply that $\eta^{f}\left(T_{n}^{\prime}, \tilde{v}\right) \rightarrow-\infty$ as $n \rightarrow \infty$. However this is impossible because $\eta^{f}(\cdot, w)$ is bounded from below for every $w \in W_{l o c}^{2,1}(0, \infty)$.

For the next lemma we need the following interpolation inequality (see e.g. Adams [1]):

Assume that $p>1$ and $\epsilon>0$. Then there exists a constant $C_{\epsilon}(p)$ such that, for every $T \geq 1$,

$$
\int_{0}^{T}\left|u^{\prime}\right|^{p} d t \leq \epsilon \int_{0}^{T}\left|u^{\prime \prime}\right|^{p} d t+C_{\epsilon}(p) \int_{0}^{T}|u|^{p} d t, \quad \forall u \in W^{2, p}(0, T)
$$

Lemma 2.2. - (i) For every $\tau>0$ there exist positive constants $b_{0}, b_{1}, b_{2}$ (depending on $\tau$ ) such that, for every $T \geq \tau$,

$$
I^{f}(0, T, v) \geq \int_{0}^{T} \frac{1}{2}\left(a_{3}\left|v^{\prime \prime}\right|^{\gamma}+a_{1}|v|^{\alpha}\right) d t-b_{0} T \geq b_{1}\|v\|_{C^{1}(0, T)}-b_{2} T,
$$

for every $v \in W^{2,1}(0, T)$ and every $f \in \overline{\mathfrak{M}}$. In particular, for every $M>0$ and $T \geq \tau$ there exists a constant $b_{\tau}(M, T)>0$ (depending continuously on $M, T$ ) such that, for every $f \in \overline{\mathfrak{M}}$,

$$
\begin{aligned}
v \in W^{2,1}(0, T), & I^{f}(0, T, v) \leq M \\
& \Longrightarrow v \in W^{2, \gamma}(0, T), \quad\|v\|_{W^{2, \gamma}(0, T)} \leq b_{\tau}(M, T) .
\end{aligned}
$$

(ii) For every $f \in \overrightarrow{\mathfrak{M}}$ : if $v \in W_{l o c}^{2,1}(0, \infty)$ is an $(f)$-good function then,

$$
\sup _{T \geq 0} \int_{T}^{T+1}\left(\left|v^{\prime \prime}\right|^{\gamma}+|v|^{\alpha}\right) d t<\infty
$$

Consequently, $v$ and ' $v^{\prime}$ are uniformly continuous on $[0, \infty)$.

Proof. - (i) In the proof we shall assume that $\tau=1$. For arbitrary $\tau>0$ the result can be obtained by rescaling. By (2.2), every $f \in \mathfrak{M}$ satisfies,

$$
f(x) \geq a_{1}\left|x_{1}\right|^{\alpha}-a_{2}\left|x_{2}\right|^{\beta}+a_{3}\left|x_{3}\right|^{\gamma}-a_{4} .
$$


Clearly this remains valid for every $f \in \overline{\mathfrak{M}}$. Note that if $\beta=1$ then $\gamma^{\prime}=\min (\alpha, \gamma)>1$ and therefore, if $\beta^{\prime} \in\left(1, \gamma^{\prime}\right)$ we have,

$$
f(x) \geq a_{1}\left|x_{1}\right|^{\alpha}-a_{2}\left|x_{2}\right|^{\beta^{\prime}}+a_{3}\left|x_{3}\right|^{\gamma}-\left(a_{2}+a_{4}\right) .
$$

Therefore, without loss of generality, we may assume that $\beta>1$. Hence, by (2.6) with $p=\beta$ and $\epsilon=\frac{a_{3}}{2 a_{2}}$, we find that, for $f \in \overline{\mathfrak{M}}$ and $T \geq 1$

$$
I^{f}(0, T, v) \geq \int_{0}^{T} \frac{1}{2}\left(a_{3}\left|v^{\prime \prime}\right|^{\gamma}+a_{1}|v|^{\alpha}\right) d t-b_{0} T, \quad \forall v \in W^{2,1}(0, T)
$$

where

$$
b_{0}=\max _{t \geq 0}\left(a_{2} C_{\epsilon}(\beta) t^{\beta}-a_{1} t^{\alpha} / 2\right)+a_{4}+a_{3} / 2 .
$$

(In fact, $v \in C^{1}[0, T]$. Therefore, by (2.2), $I^{f}(0, T ; v)$ is finite if $v^{\prime \prime} \in L^{\gamma}(0, T)$ and $+\infty$ otherwise.) This proves the first inequality in (2.7). In order to obtain the second inequality in (2.7) observe that,

$$
\begin{aligned}
\int_{s}^{s+1}\left(\left|v^{\prime \prime}\right|^{\gamma}+|v|^{\alpha}\right) d t & \geq \int_{s}^{s+1}\left(\left|v^{\prime \prime}\right|^{\gamma^{\prime}}+|v|^{\gamma^{\prime}}\right) d t-1 \\
& \geq c_{0} \sup _{s \leq t \leq s+1}\left(|v(t)|+\left|v^{\prime}(t)\right|\right)^{\gamma^{\prime}}-1,
\end{aligned}
$$

for every $s \in[0, T-1]$, where $c_{0}$ is a constant which depends only on $\gamma^{\prime}=\min (\alpha, \gamma)$. Combining this with the first inequality in (2.7) we obtain,

$$
\begin{aligned}
& I^{f}(0, T, v) \geq c_{1} \int_{0}^{T}\left(\left|v^{\prime \prime}\right|^{\gamma}+|v|^{\alpha}\right) d t-b_{0} T \\
& \quad \geq c_{1}\left(c_{0} \sup _{0 \leq t \leq T}\left(|v(t)|+\left|v^{\prime}(t)\right|\right)^{\gamma^{\prime}}-1\right)-b_{0} T \\
& \quad \geq c_{1}\left(c_{0}\|v\|_{C^{1}(0, T)}-2\right)-b_{0} T,
\end{aligned}
$$

where $c_{1}$ is a constant which depends only on $a_{1}, a_{3}$. This completes the proof of (2.7). Finally (2.8) follows from (2.7):

$$
\int_{0}^{T}\left|v^{\prime \prime}\right|^{\gamma} d t \leq 2\left(M+T b_{0}\right) / a_{3}, \quad \int_{0}^{T}|v|^{\gamma} d t \leq T\left(\left(M+b_{2}\right) / b_{1}\right)^{\gamma},
$$

for every $v$ as in (2.8).

(ii) Since $v$ is $(f)$-good, $\left(v, v^{\prime}\right)$ is bounded in $[0, \infty)$. Clearly, $U_{1}^{f}(x, y)$ is bounded for $(x, y)$ in a compact set. Therefore Lemma 2.1 implies that Vol. $16, n^{\circ} 5-1999$. 
$I^{f}(T, T+1, v)$ is bounded by a bound independent of $T \geq 0$. Hence (2.9) follows from (2.7).

Using these lemmas it is easy to verify that,

LEMMA 2.3. -For $f \in \mathfrak{M}$, (STP) implies (ATP).

Proof. - Assume that $f$ has (STP) and let $v$ be an $(f)$-good function. Pick $\xi \in \Omega(v)$ and let $\left\{t_{k}\right\}$ be a sequence tending to $+\infty$ such that $\left(v, v^{\prime}\right)\left(t_{k}\right) \rightarrow \xi$. Put $v_{k}(t)=v\left(t+t_{k}\right), t \geq-t_{k}$. By Lemma 2.2, for every bounded interval $D$,

$$
\sup _{k} \int_{D}\left(\left|v_{k}^{\prime \prime}\right|^{\gamma}+\left|v_{k}\right|^{\alpha}\right) d t<\infty .
$$

Therefore there exists a subsequence $v_{k_{n}}$ which converges weakly, say to $u$, in $W_{l o c}^{2, \gamma}\left(R^{1}\right)$. In particular $\left\{\left(v_{k_{n}}, v_{k_{n}}^{\prime}\right)\right\}$ converges uniformly on compact sets. Applying inequality (2.4) to $v_{k_{n}}$ and taking the limit, we find that (for every bounded interval $D=(0, T)$ ) $\left.u\right|_{D}$ is a minimizer of problem $\left(P_{D}^{x, y}\right)$, where $x, y$ are the values of $\left(u, u^{\prime}\right)$ at the endpoints of $D$. This is a consequence of the continuity of $U_{T}^{f}(\cdot, \cdot)$ in $R^{2}$ and of the weak lower semicontinuity of the functional $I^{f}(0, T, \cdot)$ in $W^{2, \gamma}(D)$, (see [3]). Since $f$ has (STP) it follows that, for every $\epsilon>0,(1.8)$ holds with $v$ replaced by an arbitrary translate of $u$, i.e. $u(\cdot+\tau), \tau \in R^{1}$. Consequently, if $w$ is a periodic minimizer of $\left(P_{\infty}\right)$ then, $E:=\left\{\left(u, u^{\prime}\right)(t): t \in R^{1}\right\}=\Omega(w)$. In particular, $\xi=\left(u, u^{\prime}\right)(0) \in \Omega(w)$ and we conclude that $\Omega(v) \subset \Omega(w)$. On the other hand $E \subset \Omega(v)$, so that $\Omega(v)-\Omega(w)$. Thus $f$ possesses (ATP).

The fact that (ATP) implies (STP) requires a more delicate argument. Actually we shall prove a more comprehensive result, which will also be used in the proof of Theorem 1.3. Roughly this result states that if $f \in \mathfrak{M}$ has (ATP) then, for every $\epsilon>0$ therc exists $\delta>0$ such that, if $v$ is an $(f, \delta)$-approximate minimizer in $(0, T)$ and $T$ is sufficiently large, then $v$ satisfies (1.8), which is the condition required for (STP). Furthermore this property persists in a neighborhood of $f$ in $\mathfrak{M}$. The precise formulation follows.

THEOREM 2.1. - Assume that $g \in \mathfrak{M}$ satisfies (ATP). Let $w$ be a periodic minimizer of $\left(P_{\infty}\right)$ with integrand $g$ and let $T_{w}>0$ be a period of $w$.

Given $\epsilon, M>0$ there exists a neighbourhood of $g$ in $\overline{\mathfrak{M}}$, say $\mathfrak{U}_{g}$, and positive numbers $\delta, \ell$ such that the following statement holds :

Let $f \in \mathfrak{U}_{g}$ and let $T \geq T_{w}+2 \ell$. If $v \in W^{2,1}(0, T)$ satisfies,

$$
\left|X_{v}(0)\right| \leq M,\left|X_{v}(T)\right| \leq M, \quad I^{f}(0, T, v) \leq U_{T}^{f}\left(X_{v}(0), X_{v}(T)\right)+\delta,
$$


then, for each $s \in\left[\ell, T-T_{w}-\ell\right]$ there exists $\xi \in\left[0, T_{w}\right]$ such that,

$$
\left|X_{v}(s+t)-X_{w}(\xi+t)\right| \leq \epsilon, \quad \forall t \in\left[0, T_{w}\right]
$$

Remark. - The conclusion of the theorem can be slightly strengthened as follows:

There exist $\tau_{1} \in[0, \ell]$ and $\tau_{2} \in[T-\ell, T]$ such that, for every $s \in\left[\tau_{1}, \tau_{2}-T_{w}\right]$ there exists $\xi \in\left[0, T_{w}\right]$ such that (2.14) holds. Furthermore, if

$$
\left.d\left(X_{v}(0), \Omega(w)\right) \leq \delta, \text { (respectively } d\left(X_{v}(T), \Omega(w)\right) \leq \delta\right),
$$

the statement holds with $\tau_{1}=0$, (respectively $\tau_{2}=T$ ).

The proof of the theorem will be based on several lemmas. One of the key ingredients in this proof is provided by the following result due to Leizarowitz and Mizel [10, Sec. 4]. (See also Leizarowitz [9] for a similar result in the context of a discrete model.)

Proposition 2.1. - Let $f \in \overline{\mathfrak{M}}$. Then there exist a continuous function $\pi^{f}: R^{2} \rightarrow R^{1}$ given by,

$$
\begin{gathered}
\pi^{f}(x)=\inf \left\{\liminf _{T \rightarrow \infty}\left[I^{f}(0, T, w)-T \mu(f)\right]: w \in W_{l o c}^{2,1}(0, \infty), X_{w}(0)=x\right\}, \\
x \in R^{2}
\end{gathered}
$$

and a continuous nonnegative function $(T, x, y) \rightarrow \theta_{T}^{f}(x, y)$ defined for $T>0$ and $x, y \in R^{2}$ such that,

$$
U_{T}^{f}(x, y)=T \mu(f)+\pi^{f}(x)-\pi^{f}(y)+\theta_{T}^{f}(x, y)
$$

for all $x, y, T$ as above. Furthermore, for every $T>0$ and every $x \in R^{2}$ there is $y \in R^{2}$ such that $\theta_{T}^{f}(x, y)=0$.

Let $f \in \overline{\mathfrak{M}}$. For $D=\left(T_{1}, T_{2}\right)$ and $v \in W^{2,1}(D)$ put,

$$
\begin{aligned}
& \Theta^{f}(D ; v)=\theta_{T_{2}-T_{1}}^{f}\left(X_{v}\left(T_{1}\right), X_{v}\left(T_{2}\right)\right) \\
& \Gamma^{f}(D ; v)=I^{f}\left(T_{1}, T_{2}, v\right)-\left(T_{2}-T_{1}\right) \mu(f)+\pi^{f}\left(X_{v}\left(T_{2}\right)\right)-\pi^{f}\left(X_{v}\left(T_{1}\right)\right) .
\end{aligned}
$$

From (2.15) and Proposition 2.1 it follows that

$$
\Gamma^{f}(D ; v) \geq \Theta^{f}(D ; v) \geq 0
$$

Clearly, if $v$ is a minimizer of $\left(P_{D}^{x, y}\right), x=X_{v}\left(T_{1}\right), y=X_{v}\left(T_{2}\right)$ then $\Gamma^{f}(D ; v)=\Theta^{f}(D ; v)$. However $\Gamma^{f}(D ; v)$ may be positive even in this 
case. Note that, in the present notation, a function $v \in W^{2,1}(D)$ is an $(f, \delta)$-approximate minimizer in $D$ (see (2.3b)), iff

$$
\Gamma^{f}(D ; v)-\Theta^{f}(D ; v) \leq \delta
$$

In this context we introduce the following additional terminology: Let $v$ be a minimizer of $\left(P_{\infty}\right)$. We shall say that $v$ is $(f)$-perfect if

$$
\Gamma^{f}(D, u)=0 \text { for every bounded interval } D .
$$

If $v \in W_{\text {loc }}^{2,1}(R) \cap W^{1, \infty}(R)$ and $v$ satisfies (2.15b), then $v$ is a minimizer of $\left(P_{\infty}\right)$ and hence it is $(f)$-perfect. This is an immediate consequence of the definition of $\Gamma^{f}$ and the fact that $\pi^{f}$ is continuous.

Obviously every $(f)$-perfect minimizer is c-optimal. Using this fact, it can be shown that every $(f)$-perfect minimizer is $(f)$-good (see Proposition 2.3 below). Clearly the converse does not hold, but a partial converse is provided by the following result.

LEMMA 2.4. -Let $f \in \overline{\mathfrak{M}}$ and suppose that $v$ is $(f)$-good. Then, for every $\delta>0$ there exists $T(\delta)$ such that, for $D=\left(T_{1}, T_{2}\right)$,

$$
\Gamma^{f}(D ; v) \leq \delta, \quad \forall T_{1} \geq T(\delta)
$$

In particular every periodic minimizer of $\left(P_{\infty}\right)$ is $(f)$-perfect.

Proof. - Since $\pi^{f}$ is continuous, if $v$ is an $(f)$-good function then $\Gamma^{f}(D ; v)$ is bounded. Furthermore, since $D \rightarrow \Gamma^{f}(D ; v)$ is an additive, non-negative set function, it follows that for every $\delta>0$ there exists $I^{\prime}(\delta)>0$ such that $(2.16)$ holds. The last statement of the lemma is a consequence of this inequality.

The next result shows that every $(f)$-good function generates a family of perfect minimizers.

LEMMA 2.5. - Let $f \in \overline{\mathfrak{M}}$ and let $v \in W_{\text {loc }}^{2,1}(0, \infty)$ be an $(f)$-good function. Then, given $\xi \in \Omega(v)$, there exists $u \in W_{l o c}^{2,1}\left(R^{1}\right)$ such that

$$
\left\{\left(u, u^{\prime}\right)(t): t \in R^{1}\right\} \subset \Omega(v) \text { and }\left(u, u^{\prime}\right)(0)=\xi,
$$

and $u$ is an $(f)$-perfect minimizer.

Proof. - Let $u$ be constructed as in Lemma 2.3. Then, $u$ satisfies (*) and, in the notation of that lemma,

$$
\Gamma^{f}(D, u) \leq \liminf _{k \rightarrow \infty} \Gamma^{f}\left(D+t_{n_{k}}, v\right) .
$$


This follows from the growth conditions on $f$ (see (2.2)), and the fact that $v_{n_{k}} \rightarrow u$ weakly in $W^{2, \gamma}(D)$. However, by Lemma 2.4, $\Gamma^{f}(D+\tau, v) \rightarrow 0$ as $\tau \rightarrow \infty$. Therefore $u$ satisfies (2.15b) and consequently, since $u \in W^{1, \infty}(R)$, it follows that it is $(J)$-perfect.

Another useful ingredient in our proof is the following result for which we refer the reader to [10] (proof of Proposition 4.4) and [16].

PROPOSITION 2.2. - Let $f \in \overline{\mathfrak{M}}$. For every $M_{1}, M_{2}, c>0$ there exists a positive number $A=A_{f}\left(M_{1}, M_{2}, c\right)$ such that the following statement holds for every $T \geq c$. If

$$
v \in W^{2,1}(0, T), \quad\left|X_{v}(0)\right| \leq M_{1}, \quad\left|X_{v}(T)\right| \leq M_{1},
$$

and if $v$ is an $\left(f, M_{2}\right)$-approximate minimizer in $(0, T)($ see $(2.3 b))$ then,

$$
\left|X_{v}(t)\right| \leq A, \quad \forall t \in[0, T] .
$$

Furthermore, for every $g \in \overline{\mathfrak{M}}$ there is a neighbourhood $\mathfrak{U}_{g}$ in $\overline{\mathfrak{M}}$ such that $A_{f}\left(M_{1}, M_{2}, c\right)$ can be chosen uniformly with respect to $f$ in $\mathfrak{U}_{y}$.

We also need the following lemma.

LEMMA 2.6. - Let $f \in \mathfrak{M}$. Then, for every compact set $E$ there exists a constant $M=M(E)>0$ such that, for every $T \geq 1$,

$$
U_{T}^{f}(x, y) \leq T \mu(f)+M, \quad \forall x, y \in E .
$$

Proof. - Let $w$ be a periodic minimizer of $\left(P_{\infty}\right)$ with period $T_{w}>0$. Clearly, for every $A>0$,

$$
\sup \left\{U_{T}^{f}(x, y): x, y \in E, 1 \leq T \leq A\right\}<\infty .
$$

Therefore, it is sufficient to show that there exists $M$ such that (2.17) holds for $T \geq 4 T_{w}$. Put $D=(0, T)$. Let $\tau$ be the largest integer which does not exceed $T / T_{w}$ and put $l=2^{-1}\left(T-(\tau-1) T_{w}\right)$. Let $D^{\prime}=(l, T-l)$ so that $\left|D^{\prime}\right|=(\tau-1) T_{w}$.

Given $x, y \in E$ let $v_{1}$ (resp. $v_{2}$ ) be a minimizer of problem $\left(P_{l}^{x, z}\right)$ with $z=\left(w, w^{\prime}\right)(l)$ (resp. $\left(P_{l}^{\zeta, y}\right)$ with $\left.\zeta=\left(w, w^{\prime}\right)(T-l)\right)$. Let $v \in W^{2, \gamma}(D)$ be the function given by,

$$
v(t)=\left\{\begin{array}{l}
v_{1}(t), \quad t \in(0, l) \\
w(t), \quad t \in D^{\prime} \\
v_{2}(t-T+l), \quad t \in(T-l, T)
\end{array}\right.
$$

Vol. 16, n ${ }^{\circ} 5-1999$ 
Since $w$ and $w^{\prime}$ are bounded and $T_{w} / 2 \leq l \leq T_{w}$ it follows that there exists a constant $M_{1}$ (independent of $x, y, T$ ) such that,

$$
U_{l}^{f}(x, z)=I^{f}\left(0, l, v_{1}\right) \leq M_{1} \text { and } U_{l}^{f}(\zeta, y)=I^{f}\left(0, l, v_{2}\right) \leq M_{1}
$$

Since $I^{f}(l, T-l, w)=(T-2 l) \mu(f)$ it follows that,

$$
U_{T}^{f}(x, y) \leq I^{f}(0, T, v) \leq(T-2 l) \mu(f)+2 M_{1}
$$

which implies (2.17).

Using these results we can establish the following relation between approximate minimizers and $(f)$-good functions.

Proposition 2.3. - Let $f \in \mathfrak{M}$ and $M>0$. Denote by $\mathbf{A}(f, M)$ the family of minimizers $v$ of $\left(P_{\infty}\right)$ such that $v$ is an $(f, M)$-approximate minimizer in every bounded interval $D \subset R_{+}$such that $|D| \geq 1$. Then

$$
v \in \mathbf{A}(f, M) \Longrightarrow v \text { is }(f) \text {-good }
$$

In particular, every c-optimal function is $(f)$-good. Furthermore, the family of periodic minimizers is uniformly bounded in the norm $\|v\|_{(1)}:=$ $\sup _{R_{+}}\left|X_{v}\right|$.

Proof. - Let $v$ be a minimizer of $\left(P_{\infty}\right)$. Then, for every $T>0$, $\lim _{T^{\prime} \rightarrow \infty} \frac{1}{T^{\prime}-T} I^{f}\left(T, T^{\prime}, v\right)=\mu(f)$. Hence there exists $T_{0}>T$ such that

$$
I^{f}\left(T_{0}, T_{0}+1, v\right) \leq M:=\mu(f)+1
$$

Consequently there exists a monotone sequence $\left\{T_{n}\right\}$ tending to $+\infty$ such that,

$$
I^{f}\left(T_{n}, T_{n}+1, v\right) \leq M, \quad n=1,2, \ldots
$$

By Lemma 2.2 there exists a constant $M_{1}$ (independent of $v$ ) such that,

$$
\sup \left\{\left|X_{v}(t)\right|: T_{n} \leq t \leq T_{n}+1\right\} \leq M_{1}, \quad n=1,2, \ldots
$$

Now suppose that, $v \in \mathbf{A}(f, M)$. Then inequality (*) and Proposition 2.2 imply that there exists a constant $M_{2}$ (independent of $v$ ) such that,

$$
\sup \left\{\left|X_{v}(t)\right|: T_{1} \leq t\right\} \leq M_{2}, \quad n=1,2, \ldots
$$

Thus $\left|X_{v}\right| \in L^{\infty}\left(R_{+}\right)$. (Note that in general $T_{1}$ depends on $v$ so that $\sup _{R_{+}}\left|X_{v}\right|$ may not be uniformly bounded relative to $v \in \mathbf{A}(f, M)$.) 
Further, inequality (2.3b), the boundedness of $X_{n}$, and Lemma 2.6 imply that,

$$
I^{f}(0, T, v) \leq U_{T}^{f}\left(X_{v}(0), X_{v}(T)\right)+M \leq T \mu(f)+M+M^{\prime}, \quad \forall T \geq 1 .
$$

where $M^{\prime}=M(E)$ is as in (2.17) with $E=c l\left\{X_{v}(t): t \in R_{+}\right\}$. Thus $\eta^{f}(\cdot, v)$ is bounded on $(1, \infty)$ and hence on $R_{+}$, i.e. $v$ is (f)-good.

If $v$ is a c-optimal function then, by definition, $X_{v}$ is bounded and therefore, by the previous part of the proof, $v$ is (f)-good.

Finally, if $v$ is a periodic minimizer then inequality (**) implies that $\sup _{R_{+}}\left|X_{v}\right| \leq M_{2}$, which proves the last assertion of the proposition.

The next lemma will be needed in order to establish the stability of (ATP).

LeMma 2.7. - Let $g \in \overline{\mathfrak{M}}$ and let $D=(0, T)$. For $M>0$ put,

$$
\mathfrak{V}_{M}(D)=\left\{v \in W^{2,1}(D): \int_{0}^{T^{\prime}}\left(\left|v^{\prime \prime}\right|^{\gamma}+|v|^{\alpha}\right) d t \leq M\right\} .
$$

Then for every $\epsilon, M>0$ there exists a neighbourhood $\mathfrak{N}_{g}$ of $g$ in $\overline{\mathfrak{M}}$ such that, for every $f \in \mathfrak{N}_{g}$,

$$
\left|I^{f}(0, T, v)-I^{g}(0, T, v)\right|<\epsilon, \quad \forall v \in \mathfrak{V}_{M}(D),
$$

and

$$
x, y \in R^{2},|x|,|y|<M \Longrightarrow\left|U_{T}^{f}(x, y)-U_{T}^{g}(x, y)\right|<\epsilon .
$$

The neighborhood $\mathfrak{N}_{g}$ can be chosen independently of $T$ for $T$ in compact sets of $(0, \infty)$.

Proof. - Put $M_{0}(T)=\sup \left\{\|v\|_{C^{1}[0, T]}: v \in \mathfrak{V}_{M}(D)\right\}$. By Lemma 2.2, if $T \in\left(T_{1}, T_{2}\right)$, with $0<T_{1}<T_{2}<\infty$, then $M_{1}=\sup _{T \in\left[T_{1}, T_{2}\right]}$ $M_{0}(T)<\infty$. For every $N, \delta>0$ let $B_{g}(N, \delta)=\{f \in \overline{\mathfrak{M}}:(f, g) \in$ $E(N, \delta)\}$ (see (2.1)). Now, given $\delta>0$ choose $N>2 M_{1}$ sufficiently large so that, for every $f \in B_{g}(N, \delta)$,

$$
\begin{aligned}
x \in R^{3},\left|x_{1}\right|,\left|x_{2}\right| \leq M_{1},\left|x_{3}\right| \geq N & \Longrightarrow g(x)>0, \\
& 1-2 \delta<f(x) / g(x)<1+2 \delta .
\end{aligned}
$$

Assume that $f \in B_{g}(N, \delta)$ and $v \in \mathfrak{V}_{M}(D)$. Then,

$$
\begin{aligned}
\left|I^{f}(0, T, v)-I^{g}(0, T, v)\right| & \leq \int_{E(v, N)}\left|(f-g)\left(v, v^{\prime} v^{\prime \prime}\right)\right| d t \\
& +\int_{E^{\prime}(v, N)}\left|(f-g)\left(v, v,^{\prime} v^{\prime \prime}\right)\right| d t
\end{aligned}
$$


where $E(v, N)=\left\{t \in D:\left|v^{\prime \prime}(t)\right|<N\right\}$ and $E^{\prime}(v, N)=D \backslash E(v, N)$. The first term on the right is bounded by $T \delta$ and the second by $2 \delta \int_{D}\left|g\left(v, v^{\prime}, v^{\prime \prime}\right)\right|$. The last integral is uniformly bounded for $v \in \mathfrak{V}_{M}(D)$. This follows from the inequality,

$$
|f|(x) \leq M_{f}\left(\left|x_{1}\right|+\left|x_{2}\right|\right)\left(1+\left|x_{3}\right|^{\gamma}\right), \quad \forall x \in R^{3}
$$

which, by (2.2), holds for $f \in \mathfrak{M}$ and remains valid also for $f \in \overline{\mathfrak{M}}$. Therefore, choosing $\delta$ sufficiently small so that the right hand side of (2.21) is smaller than $\epsilon$ and then choosing $N$ sufficiently large as indicated before, we obtain (2.18).

Finally, (2.19) is a consequence of (2.18) and the fact that (by Proposition 2.2) the family of minimizers of $\left(P_{D}^{x, y}\right),|x|,|y| \leq M$ is bounded by a bound independent of $f$ for $f$ in a ncighbourhood of $g$.

The next lemma plays an important role in the proof of Theorem 2.1 and the results following it.

Lemma 2.8. - Let $f \in \mathfrak{M}$ and let $D .=\left(T_{1}, T_{2}\right)$ be a bounded interval. Suppose that $w_{1}, w_{2} \in W^{2, I}(D)$ and that $\Gamma^{f}\left(D, w_{1}\right)=\Gamma^{f}\left(D, w_{2}\right)=0$. If there exists $\tau \in\left(T_{1}, T_{2}\right)$ such that $\left(w_{1}, w_{1}^{\prime}\right)(\tau)=\left(w_{2}, w_{2}^{\prime}\right)(\tau)$ then $w_{1}=w_{2}$ everywhere in $D$.

$$
\begin{aligned}
& \text { Proof. - Put } \\
& \qquad u(t)=w_{1}(t), t \in\left[T_{1}, \tau\right], \quad u(t)-w_{2}(t), t \in\left(\tau, T_{2}\right] .
\end{aligned}
$$

Evidently $u \in W^{2,1}(D)$ and $\Gamma^{f}(D, u)=0$. Since $u, w_{1}, w_{2}$ satisfy the Euler-Lagrange equation we conclude that $u=w_{1}, w_{2}$ everywhere in $D$.

To complete the proof of Theorem 2.1 we need two more auxilliary results, stated below as Lemmas $\Lambda$ and $B$. The proofs of these lemmas, which are more technical than the previous ones, will be given in Appendixes A and B respectively. In both of these lemmas we consider an integrand $f$ possessing (ATP) and study the relation between a fixed periodic minimizer of $\left(P_{\infty}\right)$, say $w$, and approximate minimizers of $\left(P_{(0, T)}\right)$. In Lemma $\mathrm{A}$ it is shown that (given $\epsilon, M>0$ ) there exists $\ell>T_{w}=$ (period of $w$ ) such that every $(f, M)$-approximate minimizer in $(0, T), T>\ell$, whose endvalues are bounded by $M$, is intermittently close to $w$ in the following sense. Every interval $D \subset(0, T),|D|=\ell$ contains a subinterval $D^{*}$ of length $T_{w}$ such that $\sup _{D^{*}}\left|X_{v}-X_{w^{*}}\right|<\epsilon$ where $w^{*}$ is a translate of $w$. In Lemma $\mathbf{B}$ it is shown that if in addition to the above, the endvalues of $v$ are sufficiently close to $\Omega(w)$ (=the limit set of $w$ ), and if $M$ is sufficiently small, then the relation described above holds in 
every subinterval $D^{*}$ of length $T_{w}$. (In general the translate $w^{*}$ will depend on $D^{*}$.) Finally, these properties persist in a neighborhood of the given integrand. The precise formulation follows.

Lemma A. -Suppose that $g \in \mathfrak{M}$ possesses (ATP). Let $w$ be a periodic minimizer of $\left(P_{\infty}\right)$ with integrand $g$ and let $T_{w}>0$ be a period of $w$. Given $M_{0}, M_{1}, \epsilon>0$ there exists an integer $q_{1} \geq 1$ and a neighbourhood $\mathfrak{U}$ of $g$ in $\mathfrak{M}$ such that the following statement holds.

Let $f \in \mathfrak{U}$ and $T \geq q_{1} T_{w}$. If $v \in W^{2,1}(0, T)$ satisfies

$$
\left|X_{v}(t)\right| \leq M_{0} \text { for } t=0, T, \quad I^{f}(0, T, v) \leq U_{T}^{f}\left(X_{v}(0), X_{v}(T)\right)+M_{1},
$$

then, for every $\tau \in\left[0, T-q_{1} T_{w}\right]$ there exist $\xi \in\left[0, T_{w}\right)$ and $s \in$ $\left[\tau, \tau+\left(q_{1}-1\right) T_{w}\right]$ such that

$$
\left|X_{v}(s+t)-X_{w}(\xi+t)\right| \leq \epsilon, \quad t \in\left[0, T_{w}\right] .
$$

Lemma B. -Let $g, w, T_{w}$ be as in Lemma A. Given $\epsilon>0$ there exist $\delta \in(0,1)$ and $Q_{0}>T_{w}$, such that for every $Q>Q_{0}$ there exists a neighbourhood $\mathfrak{U}_{Q}$ of $g$ in $\overline{\mathfrak{M}}$ such that the following statement holds.

Let $f \in \mathfrak{U}_{Q}$ and $\tau \in\left[Q_{0}, Q\right]$. If $v \in W^{2,1}(0, \tau)$ satisfies,

$d\left(X_{v}(t), \Omega(w)\right) \leq \delta$ for $t=0, \tau, \quad I^{f}(0, \tau, v) \leq U_{\tau}^{f}\left(X_{v}(0), X_{v}(\tau)\right)+\delta$,

then, for every $s \in\left[0, \tau-T_{w}\right]$ there exists $\xi \in\left[0, T_{w}\right)$ such that (2.25) holds.

Proof of Theorem 2.1. - It is sufficient to prove the theorem for all sufficiently large $M$. Therefore we may assume that

$$
M>2\left\|X_{w}\right\|_{L^{\infty}(R)}+8 .
$$

By Proposition 2.2 there exist a neighborhood of $g$ in $\overline{\mathfrak{M}}$, say $\mathfrak{N}(M)$, and a number $S>M+1$ such that for each $f \in \mathfrak{N}(M)$ and each $T \geq \inf \left\{1, T_{w}\right\}$ :

$$
\begin{aligned}
v \in & W^{2,1}(0, T), \quad\left|X_{v}(0)\right|,\left|X_{v}(T)\right| \\
& \leq M+1, \quad I^{f}(0, T, v) \leq U_{T}^{f}\left(X_{v}(0), X_{v}(T)\right)+4
\end{aligned}
$$

implies that,

$$
\left|X_{v}(t)\right| \leq S, t \in[0, T] .
$$

Given $\epsilon$ as in the theorem, there exist $\delta \in(0,1)$ and $Q_{0}>T_{w}$ such that the statement of Lemma B holds. 
By Lemma A there exist a positive integer $q_{1}$ and a neighborhood of $g$ in $\mathfrak{\mathfrak { M }}$, say $\mathfrak{N}(S, \delta)$, such that for each $f$ in this neighborhood and each $T \geq q_{1} T_{w}$ :

$$
\begin{aligned}
& v \in W^{2,1}(0, T), \quad\left|X_{v}(t)\right| \leq S+1 \text { for } t=0, T, \\
& \qquad I^{f}(0, T, v) \leq U_{T}^{f}\left(X_{v}(0), X_{v}(T)\right)+4
\end{aligned}
$$

implies that for every $\tau \in\left[0, T-q_{1} T_{w}\right]$ there exist $\xi \in\left[0, T_{w}\right)$ and $s \in\left[\tau, \tau+\left(q_{1}-1\right) T_{w}\right]$ such that

$$
\left|X_{v}(s+t)-X_{w}(\xi+t)\right| \leq \delta, \quad t \in\left[0, T_{w}\right]
$$

\section{Choose}

$$
Q_{1}>8\left(Q_{0}+q_{1} T_{w}\right)
$$

By Lemma $B$ there exists a neighborhood of $g$ in $\overline{\mathfrak{M}}$, say $\mathfrak{N}_{\epsilon}$ such that for each $f \in \mathfrak{N}_{\epsilon}$ and each $\tau \in\left[Q_{0}, Q_{1}\right]$ :

If $v \in W^{2,1}(0, \tau)$ satisfies $(2.26)$ then for every $s \in\left[0, \tau-T_{w}\right]$ there is $\xi \in\left[0, T_{w}\right)$ such that,

$$
\left|X_{v}(s+t)-X_{w}(\xi+t)\right| \leq \epsilon, \quad t \in\left[0, T_{w}\right] .
$$

We claim that the statement of the theorem holds with $\mathfrak{U}_{g}=\mathfrak{N}(M) \cap$ $\mathfrak{N}(S, \delta) \cap \mathfrak{N}_{\epsilon}$, with $\delta$ as above and $\ell=2 q_{1} T_{w}+4\left(Q_{1}+4\right)$.

Assume that $f \in \mathfrak{U}_{g}, T \geq 2 \ell+T_{w}$ and $v$ satisfies (2.13). Then $v$ satisfies (2.28) and consequently (2.29). Therefore, for each $\tau \in\left[0, T-q_{1} T_{w}\right]$ there exist $\xi \in\left[0, T_{w}\right)$ and $s \in\left[\tau, \tau+\left(q_{1}-1\right) T_{w}\right]$ such that $(2.30)$ holds. Let $m$ be the largest integer such that $(m+1) q_{1} T_{w} \leq T$. Put $\tau_{k}=k q_{1} T_{w}, k=0, \cdots, m+1$. Then, for $k=0, \cdots, m, \tau_{k}$ is in $\left[0, T-q_{1} T_{w}\right]$ and consequently there exists $\xi_{k} \in\left[0, T_{w}\right)$ and $s_{k} \in\left[\tau_{k}, \tau_{k}+\left(q_{1}-1\right) T_{w}\right] \subset\left[\tau_{k}, \tau_{k+1}\right)$ such that,

$$
\left|X_{v}\left(s_{k}+t\right)-X_{w}\left(\xi_{k}+t\right)\right| \leq \delta, \quad t \in\left[0, T_{w}\right], k=0, \cdots, m
$$

This implies,

$$
d\left(X_{v}\left(s_{k}\right), \Omega(w)\right) \leq \delta, k=0, \cdots, m
$$

Let $\nu_{0}$ be the smallest integer such that $\nu_{0} \geq Q_{0} /\left(q_{1} T_{w}\right)$ and let $\nu_{1}$ be the largest integer such that $\nu_{1} \leq Q_{1} /\left(q_{1} T_{w}\right)$. Since $Q_{1}-Q_{0}>8 q_{1} T_{w}$ we 
have $\nu_{1}-\nu_{0}>6$. an interval Put $D_{j, k}:=\left[s_{j}, s_{k}\right]$ where $0 \leq j<k \leq m$ and observe that if $\nu_{0}+1<k-j \leq \nu_{1}-1$ then,

$$
Q_{0} \leq \nu_{0} q_{1} T_{w}<\tau_{k}-\tau_{j+1} \leq\left|D_{j, k}\right| \leq \tau_{k+1}-\tau_{j} \leq \nu_{1} q_{1} T_{w} \leq Q_{1} .
$$

Further observe that the last inequality in (2.13) implies that,

$$
I^{f}\left(s_{j}, s_{k}, v\right) \leq U_{T}^{f}\left(X_{v}\left(s_{j}\right), X_{v}\left(s_{k}\right)\right)+\delta .
$$

Indeed this holds for every subinterval of $[0, T]$ because,

$$
I^{f}(a, b, v) \text { is additive and } U_{b-a}^{f}\left(X_{v}(a), X_{v}(b)\right) \text { is subadditive }
$$

on finite partitions of $(0, T)$ consisting of subintervals and because

$$
I^{f}(a, b, v) \geq U_{b-a}^{f}\left(X_{v}(a), X_{v}(b)\right) .
$$

Therefore we may apply Lemma B to the function $v$ restricted to $D_{j, k}$ where $\nu_{0}+1<k-j \leq \nu_{1}-1$, and conclude that for every $s \in\left[s_{j}, s_{k}-T_{w}\right]$ there exists $\xi \in\left[0, T_{w}\right)$ such that (2.32) holds. Finally this implies that for every $s \in\left[s_{0}, s_{m}-T_{w}\right]$ there exists $\xi \in\left[0, T_{w}\right)$ such that (2.32) holds. Since $s_{0} \leq q_{1} T_{w}$ and $T-s_{m} \geq 2 q_{1} T_{w}$, we find that the theorem holds with $\ell$ as above.

The following result is an immediate consequence of Theorem 2.1, Proposition 2.2 and Lemma 2.1. Roughly it states that if $f$ has (ATP) and $w$ is a periodic minimizer of $\left(P_{\infty}\right)$ then every $(f)$-good function is eventually 'close' to $w$.

THEOREM 2.2. - Assume that $g \in \mathfrak{M}$ has $(A T P)$ and $w \in W_{l o c}^{2,1}\left(R^{1}\right)$ is a periodic $(g)$-minimizer with a period $T_{w}>0$. Then, for every $\epsilon>0$, there exists a neighborhood $\mathfrak{U}$ of $g$ in $\overline{\mathfrak{M}}$ such that for each $f \in \mathfrak{U}$ :

If $v$ is an $(f)$-good function, there exists $t_{\epsilon}$ (depending on $\left.\epsilon, v\right)$ such that, for every $s \geq t_{\epsilon}$, there exists $\xi \in\left[0, T_{w}\right)$ such that,

$$
\left|X_{v}(s+t)-X_{w}(\xi+t)\right| \leq \epsilon, \quad t \in\left[0, T_{w}\right] .
$$

CoRollaRY 2.1. - - If $f \in \mathfrak{M}$ has (ATP) then problem $\left(P_{\infty}\right)$ possesses a unique (up to translation) periodic minimizer.

Finally we observe that Theorem 1.1 can be easily deduced from Theorem 2.1. Suppose that $G$ satisfies (1.1) and (1.2) and let $\mathfrak{L}\left(\alpha, b_{2}, b_{3}\right)$ and $\mathfrak{L}_{G}\left(\alpha, b_{2}, b_{3}\right)$ be defined as in (1.3),(1.4). Clearly, for $G$ and $\mathfrak{L}$ as in (1.1)-(1.4) and an appropriate choice of $a$,

$$
\mathfrak{L}_{G}\left(\alpha, b_{2}, b_{3}\right) \subset \mathfrak{M}(\alpha, \dot{\beta}, \gamma, a),
$$

and the operator

$$
\phi \rightarrow F_{\phi} \in \mathfrak{M}(\alpha, \beta, \gamma, a), \quad \phi \in \mathfrak{L}\left(\alpha, b_{2}, b_{3}\right)
$$

is continuous. Therefore Theorem 2.1 implies Theorem 1.1. 


\section{PROOF OF THEOREMS $1.2,1.3$}

First we establish a more general version of Theorem 1.2:

THEOREM 3.1. - Let $f \in \mathfrak{M}$. Then there exists a nonnegative function $\phi \in C^{\infty}\left(R^{1}\right)$ such that $\phi(t)>0$ for all large $|t|, \phi^{(m)}$ is bounded for every $m \geq 0$, and the following statement holds.

Denote

$$
f_{\rho}\left(x_{1}, x_{2}, x_{3}\right)=f\left(x_{1}, x_{2}, x_{3}\right)+\rho \phi\left(x_{1}\right), \quad\left(\rho, x_{1}, x_{2}, x_{3}\right) \in R^{4}
$$

Then for each $\rho \in(0,1), f_{\rho} \in \mathfrak{M}, \mu\left(f_{\rho}\right)=\mu(f)$ and problem $\left(P_{\infty}\right)$ with $f=f_{\rho}$ possesses a unique (up to translation) periodic minimizer.

We start with a brief description of the strategy of the proof, which will be presented through several lemmas. Given $f \in \mathfrak{M}$, denote by $\mathfrak{E}(f)$ the set of all periodic $(f)$-minimizers of $\left(P_{\infty}\right)$. If $w \in \mathfrak{E}(f)$ is not a constant, we denote by $\tau(w)$ the minimal period of $w$. In the first lemma we show that every non-constant periodic minimizer $w$ has precisely two extremal points in each interval $[a, a+\tau(w))$ and is strictly monotone between two consecutive extremal points. Using this fact we show that if $\mu(f)<\inf \left\{f(t, 0,0): t \in R^{1}\right\}$, then the set $\{\tau(w): w \in \mathfrak{E}(f)\}$ is bounded. Next we show that there exists $w^{*} \in \mathcal{E}(f)$ whose range $D_{w^{*}}$ is minimal in the sense that it is either disjoint from or stricty contained inthe range of any other element $w \in \mathfrak{E}(f)$, unless $w$ is a translate of $w^{*}$. Finally we observe that if there exists $\phi \in C^{\infty}(R)$ which vanishes on $D_{w}$, and is positive everywhere else, then the assertion of Theorem 3.1 holds. Since $D_{w^{*}}$ is a closed bounded interval, such a function is easily constructed.

Lemma 3.1. -Assume that $w \in \mathfrak{E}(f)$ and $w$ is not constant. Applying an appropriate translation we may assume that $w(0)=\min _{R^{1}} w$. Then there exists $\bar{\tau} \in(0, \tau(w))$ such that $w$ is strictly increasing in $[0, \bar{\tau}]$ and strictly decreasing in $[\bar{\tau}, \tau(w)]$.

Remark. - In the special case $f\left(v, v^{\prime}, v^{\prime \prime}\right)=\left|v^{\prime \prime}\right|^{2}-q\left|v^{\prime}\right|^{2}-\left(v^{2}-1\right)^{2}$, this lemma was independently established by Mizel, Peletier, Troy [14]. Their proof uses the special symmetries of the integrand.

Proof. - Let $E^{\prime}=\left\{\tau \in[0, \infty): w^{\prime}(\tau)=0\right\}$. We claim that $E \cap[0, \tau(w)]$ is a finite set. Otherwise there exists a sequence of positive numbers $\left\{t_{n}\right\}$ converging to a point $t^{*} \in[0, \tau(w)]$, such that $w^{\prime}\left(t_{n}\right)=0, n=1,2, \ldots$. By the mean value theorem, this implies that for $m=1, \cdots, 4$, there exists a sequence $\left\{t_{m, n}\right\}_{n=1}^{\infty}$ converging to $t^{*}$, such that $w^{(m)}\left(t_{m, n}\right)=0$ for 
all $n$. Therefore $w^{(m)}\left(t^{*}\right)=0, m=1, \cdots, 4$. Since $w$ satisfies the EulerLagrange equation corresponding to our variational problem this implies that $w$ is a constant, contrary to our assumption. (Note that, for $f \in \mathfrak{M}$ the Euler-Lagrange equation is a regular, fourth order equation.)

Put,

$$
\tau_{1}-\sup \left\{\tau \in E \cap[0, \tau(w)]: w^{\prime}(t) \geq 0, \forall t \in[0, \tau]\right\} .
$$

Clearly $\tau_{1} \in(0, \tau(w))$ and $w$ is strictly increasing in $\left(0, \tau_{1}\right)$. Similarly we definc

$$
\tau_{2}=\sup \left\{\tau \in E \cap\left(\tau_{1}, \tau(w)\right]: w^{\prime}(t) \leq 0, \forall t \in\left[\tau_{1}, \tau\right]\right\} .
$$

Proceeding in this manner we obtain a strictly increasing sequence $\left\{\tau_{j}: j=0, \cdots, k\right\}$ such that $\tau_{0}=0, \tau_{k}=\tau(w), w^{\prime}\left(\tau_{j}\right)=0, j=0, \cdots, k$ and $w^{\prime}$ does not change sign in each of the intervals $D_{j}=\left[\tau_{j}, \tau_{j+1}\right], j=$ $0, \cdots, k-1$. More precisely, $w$ is strictly increasing in $D_{j}$, if $j$ is even, and strictly decreasing in $D_{j}$, if $j$ is odd. Obviously $k$ is even.

Let $D_{j}^{*}$ denote the interval $\left[w\left(\tau_{j}\right), w\left(\tau_{j+1}\right)\right]$ (resp. $\left[w\left(\tau_{j+1}\right), w\left(\tau_{j}\right)\right]$ ) when $j$ is even (resp. odd).

Evidently, for each integer $j, 0 \leq j<k$ the function $t \rightarrow w(t) t \in D_{j}$ is invertible. Composing the inverse function thus obtained with the function $t \rightarrow w^{\prime}(t), t \in D_{j}$, we obtain a function $h_{j} \in C\left(D_{j}^{*}\right)$ such that $w^{\prime}(t)=h_{j}(w(t))$ for every $t \in D_{j}$.

Now we claim that for $i<j, w\left(\tau_{j}\right) \neq w\left(\tau_{i}\right)$, unless $i=0$ and $j=k$. Suppose that there exists $(i, j) \neq(0, k)$ such that $0 \leq i<j \leq k$ and $w\left(\tau_{j}\right)=w\left(\tau_{i}\right)$. Then let $u$ be the periodic function, with period $\tau_{j}-\tau_{i}$, such that $u(t)=w(t), t \in\left[\tau_{i}, \tau_{j}\right]$. Recall that $w^{\prime}\left(\tau_{m}\right)=0$ for $m=0, \cdots, k$. Hence $u \in W_{l o c}^{2,1}\left(R^{1}\right)$. Furthermore, by Lemma 2.4, $\Gamma^{f}(D ; u)=\Gamma^{f}(D ; w)=0$ in every bounded interval $D$. (Recall that the function $D \rightarrow \Gamma^{f}(D ; v)$ is additive.) 'Therefore by Lemma $2.8, u \equiv w$, which contradicts the assumption that the period of $u$ is strictly smaller than $\tau(w)$.

Next, we claim that, if $k>2$ then $D_{j}^{*} \subset D_{j-1}^{*}$ for $j=1, \cdots, k$. We verify this claim by induction. For $j=1$, we have $w(0) \leq w\left(\tau_{2}\right)<w\left(\tau_{1}\right)$. (Recall that $w(0)$ is the minimum of $w$.) Furthermore, since $k>2$, the previous argument yields $w(0)<w\left(\tau_{2}\right)<w\left(\tau_{1}\right)$. Now suppose that the claim holds for $j=1, \cdots, m-1$. To fix ideas assume that $m$ is even. Then we know that $w$ is strictly increasing in $D_{m}$ so that $w\left(\tau_{m+1}\right)>w\left(\tau_{m}\right)$. We must show that $w\left(\tau_{m+1}\right)<w\left(\tau_{m-1}\right)$. Suppose the contrary. Since, by assumption, $D_{m-1}^{*} \subset D_{m-2}^{*}$ it follows that,

$$
w\left(\tau_{m-2}\right)<w\left(\tau_{m}\right)<w\left(\tau_{m-1}\right)<w\left(\tau_{m+1}\right) .
$$

Vol. $16, n^{\circ} 5-1999$. 
Therefore the functions $h_{m-2}$ and $h_{m}$ defined in $D_{m-2}^{*}$ and $D_{m}^{*}$ respectively must intersect somewhere in $\left[w\left(\tau_{m}\right), w\left(\tau_{m-1}\right)\right]$. (Recall that both functions are non-negative in their intervals of definition and vanish at the end points of these intervals.) This means that there exist $s_{1} \in D_{m-2}$ and $s_{2} \in D_{m}$ such that $\left(w, w^{\prime}\right)\left(s_{1}\right)=\left(w, w^{\prime}\right)\left(s_{2}\right)$. However, applying once again Lemma 2.8 , the argument used before shows that this is impossible and proves our claim.

Combining the last two claims we conclude that, if $k>2$, the inclusion $D_{j}^{*} \subset D_{j-1}^{*}, j=1, \cdots, k$ is strict. But this is impossible because $w\left(\tau_{0}\right)=w\left(\tau_{k}\right)$.

Corollary 3.1. - Suppose that $f \subset \mathfrak{M}$ and that $f\left(x_{1}, x_{2}, x_{3}\right)=$ $f\left(x_{1},-x_{2}, x_{3}\right)$, for every $x \in R^{3}$. Let $w$ and $\bar{\tau}$ be as in the statement of the lemma. Then $w^{\prime}>0$ in $(0, \bar{\tau})$ and $w^{\prime}<0$ in $(\tau, \tau(w))$. Furthermore, $\bar{\tau}=\tau(w) / 2$ and $w$ is even.

Proof. - Since $f$ is even in the second argument, it follows that the function $\bar{w}$ given by $\bar{w}(t)=w(-t)$ is also a periodic minimizer. Recall that we assume that $w(0)=\min _{R} w$ so that $w^{\prime}(0)=0$. Consequently, $X_{w}(0)=X_{\bar{w}}(0)$. Hence, by Lemma $2.8, w \equiv \bar{w}$ i.e. $w$ is even. Further this implies that $w(t)=w(\tau(w)-t)$ for every real $t$. Now suppose that $s \in(0, \tau(w))$ and $w^{\prime}(s)=0$. Then $X_{w}(s)=X_{w}(\tau(w)-s)$. Using again Lemma 2.8 we deduce that $w(t)=w(t+2 s-\tau(w))$, for every $t \in R^{1}$. Thus $2 s-\tau(w)$ is a period of $w$ and therefore it must be equal to $k \tau(w)$ for some integer $k$. Since $s \in(0, \tau(m))$ it follows that $k=0$. This proves our assertion.

LeMma 3.2. -Assume that $f \in \mathfrak{M}$ satisfies the condition,

$$
\mu(f)<\inf \left\{f(t, 0,0): t \in R^{1}\right\} .
$$

Then no element of $\mathfrak{E}(f)$ is constant and

$$
\sup \{\tau(w): w \in \mathfrak{E}(f)\}<\infty .
$$

Remark. - This result was established by Marcus [13] in the special case $f\left(v, v^{\prime}, v^{\prime \prime}\right)=\left|v^{\prime \prime}\right|^{2}-\mu\left|v^{\prime}\right|^{2}+\psi(v)$, for a large class of potentials $\psi$.

Proof. Step 1 - Suppose that $\left\{T_{i}\right\}_{i=0}^{\infty}$ is a sequence of positive numbers tending to infinity, and that $\left\{w_{i}: w_{i} \in W^{2,1}\left(0, T_{i}\right)\right\}$ is a sequence of functions such that,

(i) $I^{f}\left(0, T_{i}, w_{i}\right)=T_{i} \mu(f)+\pi^{f}\left(X_{w_{i}}(0)\right)-\pi^{f}\left(X_{w_{i}}\left(T_{i}\right)\right), i=0,1,2, \ldots$

(ii) $\left\{\left|X_{w_{i}}(0)\right|\right\}_{i=0}^{\infty}$ and $\left\{\left|X_{u_{i}}\left(T_{i}\right)\right|\right\}_{i=0}^{\infty}$, are bounded,

(iii) $w_{i}^{\prime}(t) \geq 0, \quad t \in\left(0, T_{i}\right), i=0,1,2, \ldots$. 
We claim that,

$$
\mu(f)=\inf \left\{f(z, 0,0): z \in R^{1}\right\} .
$$

The same conclusion holds if in (3.4), the condition " $w_{i}^{\prime}(t) \geq 0$ " is replaced by the condition " $w_{i}^{\prime}(t) \leq 0$ ".

Assumption (3.4)(i) implies that $I^{f}\left(0, T_{i}, w_{i}\right)=U_{T_{i}}^{f}\left(X_{w_{i}}(0), X_{w_{i}}\left(T_{i}\right)\right)$ and consequently, Proposition 2.2 and assumption (3.4)(ii) imply that there exists $M>0$ such that,

$$
\sup _{t \in\left[0, T_{i}\right]}\left|X_{u_{i}}(t)\right| \leq M, \quad i=0,1,2, \ldots
$$

and

$$
\left\|w_{i}\right\|_{W^{2, \gamma}(T, T+1)} \leq M, \quad \forall T \in\left(0, T_{i}-1\right), i=0,1,2, \ldots
$$

Therefore there exits a subsequence (which we shall continue to denote by $\left\{w_{i}\right\}$ ) and a function $v \in W_{l o c}^{2, \gamma}(0, \infty)$ such that, for every $T \geq 1$,

$$
w_{i} \text {, } v \text { weakly in } W^{2, \gamma}(0, T) \text { as } i \rightarrow \infty \text {. }
$$

By the lower semicontinuity of integral functionals [3] and Proposition 2.1,

$$
I^{f}(0, T, v)=T \mu(f)+\pi^{f}\left(X_{v}(0)\right)-\pi^{f}\left(X_{v}(T)\right), \quad \forall T \geq 1 .
$$

By (3.7),

$$
\|v\|_{W^{2, \gamma}(T, T+1)} \leq M, \quad \forall T \in(1, \infty)
$$

and by (3.4)(iii), $v^{\prime} \geq 0$ in $(0, \infty)$. Consequently $v(t)$ possesses a finite limit, say $d_{0}$, and $v^{\prime}(t) \rightarrow 0$ as $t \rightarrow \infty$.

Let $v_{j}, j=0,1,2, \ldots$ be the function defined in $[0,1]$ by $v_{j}(t)=v(j+t)$. By (3.8) the sequence $\left\{v_{j}\right\}$ is bounded in $W^{2, \gamma}(0,1)$ and therefore a subsequence will converge weakly in this space to a function $u$. Clearly $u$ is the constant function $u \equiv d_{0}$. Since $I^{f}\left(0,1, v_{j}\right)=\mu(f)+$ $\pi^{f}\left(X_{v}(j)\right)-\pi^{f}\left(X_{v}(j+1)\right)$ and $X_{v}(j)$ converges, we conclude (by the lower semicontinuity of integral functionals) that $I^{f}(0,1, u)=\mu(f)$. This implies (3.5). It is obvious that the conclusion remains valid if the sign in $(3.4)$ (iii) is inverted.

Step 2. - Assume that the assertion of the lemma is not valid. Then there exists a sequence $\left\{w_{i}\right\}_{i=1}^{\infty}$ in $\mathfrak{E}(f)$ such that

$$
\tau\left(w_{i}\right) \rightarrow \infty \text { as } i \rightarrow \infty
$$

Vol. 16, $n^{\circ} 5-1999$ 
Without loss of generality we may assume that $w_{i}(0)=\min _{R} w_{i}, i=$ $1,2, \ldots$

By Lemma 3.1, for each integer $i \geq 1$ there exists a number $\tau_{i} \in\left(0, \tau\left(w_{i}\right)\right)$ such that $w_{i}$ is strictly increasing in $\left[0, \tau_{i}\right]$ and strictly decreasing in $\left[\bar{\tau}_{i}, \tau\left(w_{i}\right)\right]$. In view of (3.9) either $\bar{\tau}_{i} \rightarrow \infty$ or $\tau\left(w_{i}\right)-\bar{\tau}_{i} \rightarrow \infty$ or both. In the first case put $T_{i}=\bar{\tau}_{i}$ and $v_{i}=w_{i} \mid\left[0, \bar{\tau}_{i}\right]$; in the second case put $T_{i}=\tau\left(w_{i}\right)-\bar{\tau}_{i}$ and define $v_{i}$ in $\left[0, T_{i}\right]$ by, $v_{i}(t)=w_{i}\left(t+\bar{\tau}_{i}\right)$ for $i=1,2, \cdots$. Then the sequence $\left\{T_{i}\right\}$ tends to infinity and the sequence $\left\{v_{i}\right\}$ satisfies conditions (i), (iii) of Step 1 , possibly with a negative sign in (iii). Furthermore, by Proposition 2.3 there exists a number $S>0$ such that

$$
\sup \left\{\left|X_{v}(t)\right|: t \in R^{1}, v \in \mathfrak{E}(f)\right\} \leq S
$$

Thus the sequence $\left\{v_{i}\right\}$ satisfies also condition (ii).

Consequently, the statement established in Step 1 implies that (3.5) holds, which contradicts the assumptions of the lemma.

Lemma 3.3. - Let $f \in \mathfrak{M}$. If $w_{1}, w_{2} \in \mathfrak{E}(f)$ then the sets

$$
D_{i}:=\left\{w_{i}(t): t \in R\right\}, \quad i=1,2
$$

are either disjoint or one of them is contained in the other. Furthermore if, say, $D_{1} \subseteq D_{2}$ then either $w_{1}$ is a translate of $w_{2}$ or $D_{1}$ is contained in the interior of $D_{2}$.

Proof. - We may assume that $w_{i}(0)=\min _{R} w_{i}, i=1,2$. By Lemmas 2.8 and 2.4 , if $w_{1} \not \equiv w_{2}$, then for any two points $s_{i} \in\left(0, \tau\left(w_{i}\right)\right), i=1,2$ we have $\left(w_{1}, w_{1}^{\prime}\right)\left(s_{1}\right) \neq\left(w_{2}, w_{2}^{\prime}\right)\left(s_{2}\right)$. Therefore, if one of the two functions (say $w_{1}$ ) is a constant, then the value of this constant must be different from both the minimum and the maximum of $w_{2}$ so that our claim holds. Thus we assume that neither of the two functions is a constant. Hence, by Lemma 3.1 , there exists exactly one point $\bar{\tau}_{i}$ in $\left(0, \tau\left(w_{i}\right)\right)$ such that $w_{i}$ is strictly increasing in $\left[0, \bar{\tau}_{i}\right]$ and strictly decreasing in $\left[\bar{\tau}_{i}, \tau\left(w_{i}\right)\right]$. Consequently the function $w_{\imath}, i=1,2$ is represented in the phase plane $\left(w, w^{\prime}\right)$ by a simple closed curve $\Lambda_{i}$ consisting of two branches stretching between the points $\left(w_{i}(0), 0\right)$ and $\left(w_{i}\left(\bar{\tau}_{i}\right), 0\right)$ and $\Lambda_{1} \cap \Lambda_{2}=\emptyset$. Since $D_{i}=\left[w_{i}(0), w_{i}\left(\bar{\tau}_{i}\right)\right]$ this proves our claim.

Define

$$
\mathfrak{D}=\left\{\left\{w(t): t \in R^{1}\right\}: w \in \mathfrak{E}(f)\right\}
$$

Lemma 3.4. - Let $f \in \mathfrak{M}$. The set $\mathfrak{D}$, ordered according to set inclusion, possesses a minimal element $D_{0}$ such that, for every $D \in \mathfrak{D}$ either $D_{0} \subseteq D$ or $D_{0} \cap D=\emptyset$. 
Furthermore, if

$$
\mu(f)<\inf \left\{f(z, 0,0): z \in R^{1}\right\}
$$

then $\mathfrak{D}$ possesses only finitely many minimal elements.

Proof. - If $\mu(f)=\inf \left\{f(z, 0,0): z \in R^{1}\right\}$ then there exists a periodic minimizer which is a constant so that $\mathfrak{D}$ contains an element $D_{0}$ consisting of one point. Obviously $D_{0}$ is a minimal element of $\mathfrak{D}$. Therefore we may assume that (3.12) is valid. We claim that under this assumption,

$$
\alpha:=\inf \{|D|: D \in \mathfrak{D}\}>0
$$

and that there exists $v \in \mathfrak{E}(f)$ such that $\max v-\min v=\alpha$.

Let $\left\{w_{n}\right\}$ be a sequence in $\mathfrak{E}(f)$ such that $\alpha_{n}:=\max w_{n}-\inf w_{n} \rightarrow \alpha$. We may assume that each function $w_{n}$ attains its minimum at zero. Put $b_{n}:=\operatorname{minl}_{R} w_{n}, c_{n}:=\max _{R} w_{n}$ and $\tau_{n}:=\tau\left(w_{n}\right)$. By Lemma 3.2 the sequence of periods $\left\{\tau .\left(w_{n}\right)\right\}$ is bounded and, by Proposition 2.3, the set $\mathfrak{E}(f)$ is uniformly bounded. Therefore, by taking a subsequence if necessary, we may assume that $\left\{b_{n}\right\},\left\{c_{n}\right\}$ and $\left\{\tau_{n}\right\}$ converge. We denote their limits by $b^{*}, c^{*}, \tau^{*}$ respectively. By Lemma $2.2,\left\{w_{n}\right\}$ is bounded in $W_{l o c}^{2, \gamma}(R)$ and consequently there exists a subsequence $\left\{w_{n_{j}}\right\}$ which converges weakly in $W^{2, \gamma}(0, T)$ and strongly in $C^{1}[0, T]$, for any $T>0$. Its limit $v$ satisfies $b^{*}=v(0)=\min _{R_{+}} v$ and $c^{*}=\max _{R_{+}} v$. By the weak lower semicontinuity of the functionals, $v$ is $(f)$-perfect (see $(2.15 b)$ ). If $\tau^{*}=0$ then $b^{*}=c^{*}$, i.e. $v$ is a constant. However, by (3.12), this is impossible. Thus $\tau^{*}>0$ and $v$ is a periodic minimizer with period $\tau^{*}$. Hence $D^{*}=\left[b^{*}, c^{*}\right] \in \mathfrak{D}$ and $c^{*}-b^{*}=\alpha$. Since $v$ is not a constant $\alpha>0$. Therefore (3.13) holds and our claim is proved. In view of Lemma 3.3 this implies that $D^{*}$ is a minimal element.

In order to verify the last statement of the lemma, observe that if $D_{1}, D_{2}$ are two distinct minimal elements of $\mathfrak{D}$ then, by Lemma 3.3, $D_{1} \cap D_{2}=\emptyset$. Therefore, the uniform boundedness of $\mathfrak{E}(f)$ and (3.13) imply that the number of minimal elements is finite.

Proof of Theorem 3.1. - Let $w_{0}$ be a function in $\mathfrak{E}(f)$ such that

$$
[b, c]=\left\{w_{0}(t): \quad t \in R\right\}
$$

is a minimal element of $\mathfrak{D}$. Let $\phi$ be a function in $C^{\infty}(R)$ such that,

$$
\phi(x)=0, \forall x \in[b, c], \quad \phi(x)>0, \forall x \in R \backslash[b, c],
$$

Vol. $16, n^{\circ} 5-1999$. 
and $\phi^{(m)} \in L^{\infty}(R), m=0,1,2, \ldots$ In the present case such a function is easily constructed. In a more general context the existence of such functions was established in [2, Ch. 2, Sec.3].

With $\phi$ as above, let $\int_{\rho}$ be defined as in the statement of the theorem. Then

$$
J^{f_{\rho}}(v) \geq J^{f}(v), \quad \forall v \in W_{l o c}^{2,1}(0, \infty) .
$$

If $v$ is a periodic function, equality holds in (3.14) if and only if

$$
\{v(t): t \in[0, \infty)\} \subseteq[b, c]
$$

Hence

$$
\mu\left(f_{\rho}\right) \geq \mu(f)=J^{f}\left(w_{0}\right)=J^{f_{\rho}}\left(w_{0}\right) \geq \mu\left(f_{\rho}\right) .
$$

Consequently, $\mu(f)=\mu\left(f_{\rho}\right)$ and $w_{0}$ is a minimizer of $\left(P_{\infty}\right)$ with integrand $f_{\rho}$. We claim that $w_{0}$ is the unique (up to translation) periodic minimizer of this problem. Indeed, if $w$ is another periodic minimizer of this problem then, by (3.14), (3.15), $w \in \mathfrak{E}(f)$ and $\{w(t): t \in R\} \subseteq[b, c]$. Since $[b, c]$ is a minimal element of $\mathfrak{D}$ it follows that $\{w(t): t \in R\}=[b, c]$. However, by Lemma 3.3, this implies that $w$ is a translate of $w_{0}$.

Next we prove a slightly stronger formulation of Theorem 1.3 (i):

TheOREM 3.2. - Let $f \in \mathfrak{M}$. If $\phi \in C^{\infty}(R)$ and $f_{\rho}$ are as in Theorem 3.1 then, for each $\rho \in(0,1)$, $f_{\rho}$ possesses $(A T P)$.

Proof. - First suppose that $\mu(f)<\inf _{R} f(\cdot, 0,0)$. In this case the statement of the theorem is an immediate consequence of Theorem 3.1 and the following result of Zaslavski [18]:

Assume that $h \in \mathfrak{M}$ and that $\mu(h)<\inf _{R} h(\cdot, 0,0)$. Then $h$ has (ATP) if and only if there exists a unique (up to translation) periodic $(h)$-minimizer.

Next suppose that $\mu(f)=\inf _{R} f(\cdot, 0,0)$. Then there exists $\xi_{0} \in R^{1}$ such that $f\left(\xi_{0}, 0,0\right)=\mu(f)$ and $\phi$ is positive everywhere except at $\xi_{0}$. By Theorem 3.1 , for every $\rho \in(0,1)$, problem $\left(P_{\infty}\right)$ with integrand $f_{\rho}$ has a unique periodic minimizer, namely the constant function with value $\xi_{0}$. In order to prove that $\left(f_{\rho}\right)$ possesses (ATP) we must prove that,

$$
\text { 16) } v \in W_{l o c}^{2,1}(0, \infty) \text { and } v \text { is }\left(f_{\rho}\right)-\operatorname{good} \Longrightarrow \lim _{t \rightarrow \infty}\left(v, v^{\prime}\right)(t)=\left(\xi_{0}, 0\right) \text {. }
$$

Let $v$ satisfy the assumptions of (3.16) for some $\rho \in(0,1)$. Then, in view of (3.14), $J^{f}(v)=\mu(f)$. Since

$$
0 \leq \eta^{f_{\rho}}(T, v)-\eta^{f}(T, v)=\int_{0}^{T} \rho \phi(v(t)) d t
$$


and $\eta^{f_{\rho}}(\cdot, v)$ is bounded on $(0, \infty)$ it follows that $\eta^{f}(\cdot, v)$ is bounded, i.e. $v$ is an $(f)$-good function, and $\lim _{T \rightarrow \infty} \int_{0}^{T} \phi(v(t)) d t<\infty$. We claim that

$$
\lim _{t \rightarrow \infty} v(t)=\xi_{0} \text {. }
$$

Indeed by Lemma $2.2 v$ and $v^{\prime}$ are uniformly continuous on $(0, \infty)$. Therefore, if there exists a sequence $\left\{t_{n}\right\}$ tending to infinity such that $v\left(t_{n}\right) \rightarrow \xi_{1} \neq \xi_{0}$ then there exists a positive $\delta$ such that

$$
\liminf _{n \rightarrow \infty} \operatorname{dist}\left(\xi_{0},\left\{v(t): t_{n}-\delta \leq t \leq t_{n}+\delta\right\}\right)>0 .
$$

Since $v$ is bounded and $\phi$ is positive except at $\xi_{0}$ this contradicts the integrability of $\phi(v(\cdot))$ on $(0, \infty)$.

Next we claim that $\lim _{t \rightarrow \infty} v^{\prime}(t)=0$. If not, assume for instance that $\lim \sup v^{\prime}(t)=\zeta>0$. Then, because of the uniform continuity of $v^{\prime}$, it follows that there exists a sequence $\left\{t_{n}\right\}$ tending to infinity and a positive $\delta$ such that $\inf \left\{v^{\prime}(t): t_{n}-\delta \leq t \leq t_{n}+\delta\right\}>\zeta / 2$ for all sufficiently large $n$. Therefore $v\left(t_{n}+\delta\right)-v\left(t_{n}\right)>\delta \zeta / 2$ for all sufficiently large $n$, which contradicts (3.17). Thus $\lim _{t \rightarrow \infty}\left(v, v^{\prime}\right)(t)=\left(\xi_{0}, 0\right)$ and (3.16) is proved.

Finally we turn to,

Proof of Theorem 1.3 (ii). - Denote by $E$ the set of all functions $\phi \in \mathfrak{L}\left(\alpha, b_{2}, b_{3}\right)$ such that $F_{\phi}$ has (ATP). By Theorem 3.2 the set $E$ is everywhere dense in $\mathfrak{L}\left(\alpha, b_{2}, b_{3}\right)$. For each $\phi \in E$ there exist $v_{\phi} \in W_{l o c}^{2,1}\left(R^{1}\right), T_{\phi}>0$ such that

$$
v_{\phi}\left(t+T_{\phi}\right)=v_{\phi}(t), t \in R^{1}, I^{F_{\phi}}\left(0, T_{\phi}, v_{\phi}\right)=\mu\left(F_{\phi}\right) T_{\phi} .
$$

Let $\phi \in E, n \geq 1$ be an integer. By (3.18), the definition of the set $E$, the continuity of the operator

$$
\phi \rightarrow F_{\phi}, \quad \phi \in \mathfrak{L}\left(\alpha, b_{2}, b_{3}\right) .
$$

and Theorem 2.2 there exist an open neighborhood $U(\phi, n)$ of $\phi$ in $\mathfrak{L}\left(\alpha, b_{2}, b_{3}\right)$ such that for each $\psi \in U(\phi, n)$ and each $\left(F_{\psi}\right)$-good function $w \in W_{l o c}^{2,1}(0, \infty)$

$$
\operatorname{dist}\left(\Omega(w),\left\{X_{v_{\phi}}(t): t \in R^{1}\right\}\right) \leq(2 n)^{1} .
$$

Define

$$
\mathfrak{F}=\cap_{n=1}^{\infty} \cup\{U(\phi, n): \phi \in E\} .
$$

Let $h \in \mathfrak{F}, w_{1}, w_{2}$ be $\left(F_{h}\right)$-good functions. To complete the proof of the theorem it is sufficient to show that $\Omega\left(w_{1}\right)=\Omega\left(w_{2}\right)$. Let $\epsilon \in(0,1)$. There exist an integer $n \geq 8 \epsilon^{-1}$ and $\phi \in E$ such that $h \in U(\phi, n)$. It follows from the definition of $U(\phi, n)$ that

$$
\operatorname{dist}\left(\Omega\left(w_{i}\right),\left\{X_{v_{\phi}}(t): t \in R^{1}\right\}\right) \leq(2 n)^{-1}, i=1,2, \operatorname{dist}\left(\Omega\left(w_{1}\right), \Omega\left(w_{2}\right)\right) \leq \epsilon .
$$

This completes the proof of the theorem.

Vol, 16, n $n^{\circ}$ 5-1999. 


\section{APPENDIX A}

This appendix is devoted to the proof of Lemma A, which will be based on several additional lemmas.

LEMmA A.1. - Let $\epsilon, M>0$. Then there exist $\delta>0$ and an integer $q_{1} \geq 1$ such that for each $v \in W^{2,1}\left(0, q_{1} T_{w}\right)$ which satisfy

$$
\begin{gathered}
\left|X_{v}(s)\right| \leq M, \quad s=0, q_{1} T_{w}, \\
I^{g}\left(0, q_{1} T_{w}, v\right) \leq q_{1} T_{w} \mu(g)+\pi^{g}\left(X_{v}(0)\right)-\pi^{g}\left(X_{v}\left(q_{1} T_{w}\right)\right)+\delta
\end{gathered}
$$

there exist $\xi \in\left[0, T_{w}\right), \tau \in\left[0,\left(q_{1}-1\right) T_{w}\right]$ such that

$$
\left|X_{v}(\tau+t)-X_{w}(\xi+t)\right| \leq \epsilon, \quad t \in\left[0, T_{u}\right] .
$$

Proof. - Let us assume the converse. Then for each integer $p \geq 1$ there exists $v_{p} \in W^{2,1}\left(0, p^{\prime} T_{w}\right)$ such that

$$
\left|X_{v_{p}}(s)\right| \leq M, \quad s=0, p T_{u},
$$

$$
I^{g}\left(0, p T_{w}, v_{p}\right) \leq p T_{w} \mu(g)+\pi^{g}\left(X_{v_{p}}(0)\right)-\pi^{g}\left(X_{v_{p}}\left(p T_{w}\right)\right)+2^{-p}
$$

and for each $\xi \in\left[0, T_{w}\right)$, each $\tau \in\left[0,(p-1) T_{w}\right]$

$$
\sup \left\{\left|X_{v_{p}}(\tau+t)-X_{w}(\xi+t)\right|: t \in\left[0, T_{w}\right]\right\}>\epsilon .
$$

By (A.2) and Proposition 2.2 there exists $M_{1}>0$ such that for each integer $p \geq 1$

$$
\left|X_{v_{p}}(t)\right| \leq M_{1}, \quad t \in\left[0, p T_{w}\right] .
$$

(A.2), (A.4) and (2.2) imply that for any integer $n \geq 1$ the sequence $\left\{v_{p}^{\prime \prime}\right\}_{p=n}^{\infty}$ is bounded in $L^{\gamma}\left[0, n T_{w}\right]$. It is easy to verify that there are $v \in W_{l o c}^{2, \gamma}(0, \infty)$ and a strictly increasing subsequence of natural numbers $\left\{p_{k}\right\}_{k=1}^{\infty}$ such that for every integer $n \geq 1$

$$
v_{p_{k}} \rightarrow v \text { as } k \rightarrow \infty \text { weakly in } W^{2, \gamma}\left(0, n T_{w}\right) .
$$

By (A.2) and the lower semicontinuity of integral functionals [3] for each integer $n \geq 1$

$$
I^{g}\left(0, n T_{w}, v\right)=n T_{w} \mu(g)+\pi^{g}\left(X_{v}(0)\right)-\pi^{g}\left(X_{v}\left(n T_{w}\right)\right) .
$$


Clearly

$$
\left|X_{v}(t)\right| \leq M_{1}, \quad t \in[0, \infty) .
$$

It follows from (A.5) and the definition of $\left\{v_{p}\right\}_{p=1}^{\infty}$ (see (A.2), (A.3)) that for each $\tau \in[0, \infty)$ and each $\xi \in\left[0, T_{w}\right)$

$$
\sup \left\{\left|X_{v}(\tau+t)-X_{w}(\xi+t)\right|: t \in\left[0, T_{w}\right]\right\}>2^{-1} \epsilon .
$$

(A.6) and (A.7) imply that the function $v$ is $(g)$-good. Then

$$
\Omega(v)=\Omega(u n)
$$

There exists a sequence of numbers $\left\{t_{j}\right\}_{j=1}^{\infty} \subset(0, \infty)$ such that (A.10)

$t_{1} \geq 8 T_{w}+8, t_{j+1}-t_{j} \geq 8 T_{w}, j=1,2, \ldots, X_{v}\left(t_{j}\right) \rightarrow X_{w}(0)$ as $j \rightarrow \infty$.

For each integer $j \geq 1$ we define $u_{j} \in W^{2,1}\left(-4 T_{w}, 4 T_{w}\right)$ as follows

$$
u_{j}(t)=v\left(t_{j}+t\right), \quad t \in\left[-4 T_{w}, 4 T_{w}\right] .
$$

By (2.2), (A.11), (A.6) and (A.7) the sequence $\left\{u_{j}^{\prime \prime}\right\}_{j=1}^{\infty}$ is bounded in $L^{\gamma}\left[-4 T_{w}, 4 T_{w}\right]$. It is easy to verify that there are $u \in W^{2,1}\left(-4 T_{w}, 4 T_{w}\right)$ and a strictly increasing subsequence of natural numbers $\left\{j_{p}\right\}_{p=1}^{\infty}$ such that (A.12)

$$
\begin{gathered}
u_{j_{p}}(t) \rightarrow u(t), u_{j_{p}}^{\prime}(t) \rightarrow u^{\prime}(t) \text { as } p \rightarrow \infty \text { uniformly in }\left[-4 T_{w}, 4 T_{w}\right], \\
u_{j_{p}}^{\prime \prime} \rightarrow u^{\prime \prime} \text { as } p \rightarrow \infty \text { weakly in } L^{\gamma}\left[-4 T_{w}, 4 T_{w}\right] .
\end{gathered}
$$

By (A.6) and the lower semicontinuity of integral functionals [3]

(A.13) $I^{g}\left(-4 T_{w}, 4 T_{w}, u\right)=8 T_{w} \mu(g)+\pi^{g}\left(X_{u}\left(-4 T_{w}\right)\right)+\pi^{g}\left(X_{u}\left(4 T_{w}\right)\right)$.

Clearly

$$
X_{u}(0)=X_{w}(0)
$$

It follows from (A.11), (A.12) and (A.8) which holds for each $\tau \in[0, \infty)$ and each $\xi \in\left[0, T_{w}\right)$, that

$$
\sup \left\{\left|X_{u}(t)-X_{w}(t)\right|: t \in\left[0, T_{w}\right]\right\}>4^{-1} \epsilon .
$$

On the other hand (A.13), (A.14) and Lemma 2.8 imply that $u(t)=w(t)$ for all $t \in\left[-4 T_{w}, 4 T_{w}\right]$. The obtained contradiction proves the lemma. 
LEMmA A.2. - Let $M_{0}, M_{1}, \epsilon>0$. Then there exists an integer $q \geq 1$ such that for each $v \in W^{2.1}\left(0, q T_{w}\right)$ which satisfies

(A.15)

$\left|X_{v}(s)\right| \leq M_{0}, s=0 . q T_{w} . I^{g}\left(0, q T_{w}, v\right) \leq U_{q T_{w}}^{q}\left(X_{v}(0) \cdot X_{v}\left(q T_{w}\right)\right)+M_{1}$

there exist $\xi \in\left[0, T_{w}\right), \tau \in\left[0,(q-1) T_{w}\right]$ such that

$$
\left|X_{w}(\tau+t)-X_{w}(\xi+t)\right| \leq \epsilon . \quad t \in\left[0, T_{w}\right] .
$$

Proof. - By Proposition 2.2 there is $S_{0}>M_{0}+M_{1}+2$ such that for each $\tau \geq 2^{-1} \inf \left\{T_{w}, 1\right\}$, each $v \in W^{2,1}(0, \tau)$ which satisfies

$$
\left|X_{v}(0)\right|,\left|X_{v}(\tau)\right| \leq M_{0}, \quad I^{g}(0, \tau, v) \leq U_{\tau}^{g}\left(X_{v}(0), X_{v}(\tau)\right)+M_{1}+1
$$

the following relation holds

$$
\left|X_{1}(t)\right| \leq S_{0}, \quad t \in[0, \tau]
$$

By Lemma A.l there exists an integer $q_{1} \geq 1$ and a number $\delta>0$ such that for each $v \in W^{2,1}\left(0, q_{1} T_{w}\right)$ which satisfies

$$
\left|X_{v}(t)\right| \leq S_{0}, \quad t=0 . q_{1} T_{u},
$$

$$
I^{g}\left(0, q_{1} T_{u}, v\right) \leq q_{1} T_{w} \mu(g)+\pi^{g}\left(X_{v}(0)\right)-\pi^{g}\left(X_{v}\left(q_{1} T_{w}\right)\right)+\delta
$$

there exist $\xi \in\left[0, T_{w}\right), \tau \in\left[0,\left(q_{1}-1\right) T_{w}\right]$ such that (A.16) holds. By Lemma 2.6 there exists $K_{0}>0$ such that for each $\tau \geq 4 T_{w}$, each $x, y \in R^{2}$ satisfying $|x|,|y| \leq M_{0}+S_{0}+1$ the following relation holds

$$
U_{\tau}^{g}(x, y) \leq \tau \mu(g)+\pi^{g}(x)-\pi^{g}(y)+K_{0} .
$$

Here we use the fact that $\pi^{g}$ is bounded on compact sets. Fix an integer

$$
q>\left[\left(M_{1}+K_{0}+1\right) \delta^{-1}+4\right] q_{1} .
$$

Assume that $v \in W^{2,1}\left(0, q T_{w}\right)$ and (A.15) holds. It follows from (A.15) and the definition of $K_{0}$ (see (A.19)) that

$$
\begin{gathered}
I^{g}\left(0, q T_{w}, v\right) \leq U_{q T_{w}}^{g}\left(X_{v}(0), X_{v}\left(q T_{w}\right)\right)+M_{1} \\
\leq q T_{w} \mu(g)+\pi^{g}\left(X_{v}(0)\right)-\pi^{g}\left(X_{v}\left(q T_{w}\right)\right)+M_{1}+K_{0} .
\end{gathered}
$$

By the definition of $S_{0}$ (see (A.17)) and (A.15)

$$
\left|X_{v}(t)\right| \leq S_{0}, \quad t \in\left[0, q T_{w}\right]
$$


There exists a sequence $\left\{t_{i}\right\}_{i=0}^{s} \subset\left[0, q T_{w}\right]$ such that

(A.23) $t_{0}=0, t_{i+1}=t_{i}+q_{1} T_{w}$ if $0 \leq i \leq s-1, t_{s} \in\left[q T_{w}-q_{1} T_{w}, q T_{w}\right]$.

\section{Clearly}

$$
s \geq q q_{1}^{-1}-1 \geq 3+\delta^{-1}\left(M_{1}+K_{0}+1\right) .
$$

Together with (A.21) this implies that there is $j \in\{0, \ldots s-1\}$ for which (A.25) $I^{g}\left(t_{j}, t_{j+1}, v\right) \leq\left(t_{j+1}-t_{j}\right) \mu(g)+\pi^{g}\left(X_{v}\left(t_{j}\right)\right)-\pi^{g}\left(X_{v}\left(t_{j+1}\right)\right)+\delta$.

It follows from this relation, (A.22), (A.23) and the definition of $\delta, q_{1}$ (see (A.18)) that there exist $\xi \in\left[0, T_{w}\right), \tau \in\left[t_{j}, t_{j+1}-T_{w}\right]$ such that (A.16) holds. This completes the proof of the lemma.

Proof of Lemma A. - By Proposition 2.2 there are a neighborhood $\mathfrak{U}_{1}$ of $g$ in $\mathfrak{M}$ and a number $M_{2}>M_{0}+M_{1}$ such that for each $f \in \mathfrak{U}_{1}$, each $T \geq \inf \left\{T_{w}, 1\right\}$ and each $v \in W^{2,1}(0, T)$ satisfying (2.24) the following relation holds

$$
\left|X_{v}(t)\right| \leq M_{2}, \quad t \in[0, T]
$$

By Lemma A.2 there exists an integer $q_{1} \geq 1$ such that for each $v \in W^{2,1}\left(0, q_{1} T_{v}\right)$ which satisfies

$$
\begin{gathered}
\left|X_{v}(0)\right|,\left|X_{v}\left(q_{1} T_{w}\right)\right| \leq M_{2}, \\
I^{g}\left(0, q_{1} T_{w}, v\right) \leq U_{q_{1} T_{w}}^{g}\left(X_{v}(0), X_{v}\left(q_{1} T_{w}\right)\right)+2 M_{1}+8
\end{gathered}
$$

there exist $\xi \in\left[0, T_{w}\right), s \in\left[0,\left(q_{1}-1\right) T_{w}\right]$ such that $(2.25)$ holds.

There exists a number $\Gamma_{0}>0$ for which

$$
\sup \left\{\left|U_{q_{1} T_{w}}^{g}(x, y)\right|: x, y \in R^{2},|x|,|y| \leq M_{2}\right\} \leq \Gamma_{0} .
$$

By Lemma 2.7 there exists a neighborhood $\mathfrak{U}_{2}$ of $g$ in $\overline{\mathfrak{M}}$ such that for each $f \in \mathfrak{U}_{2}$, each $x, y \in R^{2}$ satisfying $|x|,|y| \leq M_{2}$ the relation $\left|U_{q_{1} T_{w}}^{f}(x, y)-U_{q_{1} T_{w}}^{g}(x, y)\right| \leq 2^{1}$ holds.

By Lemma 2.7 there exists a neighborhood $\mathfrak{U}_{3}$ of $g$ in $\mathfrak{M}$ such that for each $f \in \mathfrak{U}_{3}$, each $v \in W^{2,1}\left(0, q_{1} T_{w}\right)$ satisfying

$$
\inf \left\{I^{f}\left(0, q_{1} T_{w}, v\right), I^{g}\left(0, q_{1} T_{w}, v\right)\right\} \leq 2 \Gamma_{0}+2 M_{1}+4
$$

the relation $\left|I^{f}\left(0, q_{1} T_{w}, v\right)-I^{g}\left(0, q_{1} T_{w}, v\right)\right| \leq 2^{-1}$ holds. Set $\mathfrak{U}=$ $\mathfrak{U}_{1} \cap \mathfrak{U}_{2} \cap \mathfrak{U}_{3}$. 
Assume that $f \in \mathscr{U}, T \geq q_{1} T_{w}, v \in W^{2,1}(0, T)$ satisfies (2.24) and $\tau \in\left[0, T-q_{1} T_{w}\right]$. By the definition of $\mathfrak{U}_{1}$ and $M_{2}$ relation (A.26) holds. It follows from (2.24), (A.26), the definition of $\mathfrak{U}_{2}$ and (A.28) that

$$
\begin{array}{r}
I^{f}\left(\tau, \tau+q_{1} T_{u}, v\right) \leq U_{q_{1} T_{w}}^{f}\left(X_{v}(\tau), X_{v}\left(\tau+q_{1} T_{w}\right)\right)+M_{1} \\
\leq U_{q_{1} T_{w}}^{g}\left(X_{v}(\tau), X_{v}\left(\tau+q_{1} T_{w}\right)\right)+2^{-1}+M_{1} \leq \Gamma_{0}+2^{-1}+M_{1} .
\end{array}
$$

By this relation and the definition of $\mathfrak{U}_{3}$

$$
\begin{gathered}
\left|I^{f}\left(\tau, \tau+q_{1} T_{w}, v\right)-I^{g}\left(\tau, \tau+q_{1} T_{w}, v\right)\right| \leq 2^{-1}, \\
I^{g}\left(\tau, \tau+q_{1} T_{w}, v\right) \leq U_{q_{1} T_{w}}^{y}\left(X_{v}(\tau), X_{v}\left(\tau+q_{1} T_{w}\right)\right)+1+M_{1} .
\end{gathered}
$$

It follows from this relation, (A.26) and the definition of $q_{1}$ (see (A.27)) that there exist $\xi \in\left[0, T_{w}\right), s \in\left[\tau, \tau+q_{1} T_{w}-T_{w}\right]$ such that (2.25) holds. The lemma is proved.

\section{APPENDIX B}

Here we establish Lemma B whose proof is based on several auxilliary results.

The following lemma shows that given $\epsilon>0$ and a $(g)$-good function $v$, for sufficiently large $T$ the restriction of $\left(v, v^{\prime}\right)$ to $\left[T, T+T_{w}\right]$ is within $\epsilon$ of a translation of $\left(w, w^{\prime}\right)$.

LEMMA B.1. Assume that $v \in W_{\text {loc }}^{2,1}(0, \infty)$ is a $(g)$-good function and $\epsilon>0$. Then there exists $T(\epsilon)>0$ such that for each $T \geq T(\epsilon)$ there is $\xi \in\left[0, T_{w}\right)$ such that

$$
\left|X_{v}(T+t)-X_{u}(\xi+t)\right| \leq \epsilon, \quad t \in\left[0, T_{w}\right]
$$

Proof. - Since $v$ is a $(g)$-good function for each $\delta>0$ there exists $T(\delta)>0$ such that

$$
I^{g}\left(\tau_{1}, \tau_{2}, v\right) \leq\left(\tau_{2}-\tau_{1}\right) \mu(g)+\pi^{g}\left(X_{v}\left(\tau_{1}\right)\right)-\pi^{g}\left(X_{v}\left(\tau_{2}\right)\right)+\delta
$$

for each $\tau_{1} \geq I^{\prime}(\delta)$ and each $\tau_{2}>\tau_{1}$ (see Lemma 2.4).

Assume that the lemma is wrong. Then there exists a sequence of numbers $\left\{t_{i}\right\}_{i=1}^{\infty} \subset(0, \infty)$ such that

$$
t_{i} \geq T\left(2^{-i}\right)+2 i+2, \quad i=1,2, \ldots
$$


and for each integer $i \geq 1$ and each $\xi \in\left[0, T_{w}\right)$

$$
\sup \left\{\left|X_{v}\left(t_{i}+t\right)-X_{w}(\xi+t)\right|: t \in\left[0, T_{w}\right]\right\}>\epsilon .
$$

For each integer $i \geq 1$ we define $u_{i} \in W_{l o c}^{2,1}\left(-t_{i}, \infty\right)$ as follows

$$
u_{i}(t)=v\left(t_{i}+t\right), \quad t \in\left[-t_{i}, \infty\right)
$$

It follows from the definition of $T(\delta), \delta>0$ (see (B.1)), (B.2), (B.4) and (2.2) that for any integer $n \geq 1$ the sequence $\left\{u_{i}^{\prime \prime}\right\}_{i=n}^{\infty}$ is bounded in $L^{\gamma}[-n, n]$.

It is easy to see that there exist $u \in W_{\text {loc }}^{2, \gamma}\left(R^{1}\right)$ and a strictly increasing subsequence of natural numbers $\left\{i_{p}\right\}_{p=1}^{\infty}$ such that for every integer $n \geq 1$

$$
u_{i_{p}} \rightarrow u \text { as } p \rightarrow \infty \text { weakly in } W^{2, \gamma}(-n, n) .
$$

By the definition of $T(\delta), \delta>0$ (see (B.1)), (B.2), (B.4), (B.5) and the lower semicontinuity of integral functionals [3]

$$
I^{g}\left(\tau_{1}, \tau_{2}, u\right)=\left(\tau_{2}-\tau_{1}\right) \mu(g)+\pi^{g}\left(X_{u}\left(\tau_{1}\right)\right)-\pi^{g}\left(X_{u}\left(\tau_{2}\right)\right)
$$

for each $\tau_{1} \in R^{1}, \tau_{2}>\tau_{1}$.

It is easy to see that for each $t \in R^{1}$

$$
X_{u}(t) \in \Omega(v)=\left\{X_{w}(s): s \in R^{1}\right\} .
$$

Together with (B.6), Lemma 2.8 this implies that there exists $\xi_{0} \in\left[0, T_{w}\right.$ ) such that $u(t)=w\left(t+\xi_{0}\right), t \in R^{1}$. It follows from this relation and (B.5), (B.4) that there exists an integer $p_{0} \geq 1$ such that for each integer $p \geq p_{0}$

$$
\left|X_{v}\left(t_{i_{p}}+t\right)-X_{w}\left(\xi_{0}+t\right)\right| \leq 2^{-1} \epsilon, \quad t \in\left[0, T_{w}\right] .
$$

This is contradictory to the definition of $\left\{t_{i}\right\}_{i=1}^{\infty}$ (see (B.3)). The obtained contradiction proves the lemma.

LEMMA B.2. - Let $\epsilon>0$. Then there exists $\delta>0$ such that for each $\tau \geq T_{w}$ and each $s \in\left[0, \tau-T_{w}\right]$, if $v$ is a function in $W^{2,1}(0, \tau)$ such that

$$
\begin{gathered}
d\left(X_{v}(s),\left\{X_{w}(t): t \in R^{1}\right\}\right) \leq \delta, \quad s=0, \tau, \\
I^{g}(0, \tau, v) \leq \tau \mu(g)+\pi^{g}\left(X_{v}(0)\right)-\pi^{g}\left(X_{v}(\tau)\right)+\delta
\end{gathered}
$$

then there is $\xi \in\left[0, T_{w}\right)$ for which

$$
\left|X_{v}(s+t)-X_{w}(\xi+t)\right| \leq \epsilon, \quad t \in\left[0, T_{w}\right] .
$$

Vol. $16, \mathrm{n}^{\circ}$ 5-1999. 
Proof - By Proposition 2.1 and the continuity of $\pi^{g}, U_{T_{w}}^{g}$ for each integer $i \geq 1$ there exists $\delta_{i} \in\left(0,4^{-i}\right)$ such that for each $x, y \in R^{2}$ satisfying $|x-y| \leq \delta_{i}, d\left(x,\left\{X_{w}(t): t \in R^{1}\right\}\right) \leq \delta_{i}$ the following relation holds

$$
U_{T_{w}}^{g}(x, y) \leq \pi^{g}(x)-\pi^{g}(y)+T_{w} \mu(g)+2^{-i} .
$$

Assume that the lemma is wrong. Then for each integer $i \geq 1$ there exist $\tau_{i} \geq T_{w}, v_{i} \in W^{2,1}\left(0, \tau_{i}\right)$ such that

$$
\begin{gathered}
d\left(X_{v_{i}}(s),\left\{X_{w}(t): t \in R^{1}\right\}\right) \leq \delta_{i}, s=0, \tau_{i}, \\
I^{g}\left(0, \tau_{i}, v_{i}\right) \leq \tau_{i} \mu(g)+\pi^{g}\left(X_{v_{i}}(0)\right)-\pi^{g}\left(X_{v_{i}}\left(\tau_{i}\right)\right)+\delta_{i}
\end{gathered}
$$

and there exists $s_{i} \in\left[0, \tau_{i}-T_{w}\right]$ such that for each $\xi \in\left[0, T_{w}\right)$

$$
\sup \left\{\left|X_{v_{i}}\left(s_{i}+t\right)-X_{w}(\xi+t)\right|: t \in\left[0, T_{w}\right]\right\}>\epsilon .
$$

For each integer $i \geq 1$ there exist $\xi_{i}^{1}, \xi_{i}^{2} \in\left[0, T_{w}\right)$ such that

$$
\left|X_{v_{i}}(0)-X_{w}\left(\xi_{i}^{1}\right)\right|,\left|X_{v_{i}}\left(\tau_{i}\right)-X_{u}\left(\xi_{i}^{2}\right)\right| \leq \delta_{i} .
$$

For each integer $i \geq 1$ there exists a function $u_{i} \in W^{2,1}\left(0, \tau_{i}+2 T_{w}\right)$ such that

$$
\begin{aligned}
& X_{u_{i}}(0)=X_{w}\left(\xi_{i}^{1}\right), u_{i}(t)=v_{i}\left(t-T_{w}\right), t \in\left[T_{w}, T_{w}+\tau_{i}\right], X_{u_{i}}\left(\tau_{i}+2 T_{w}\right) \\
& =X_{w}\left(\xi_{i}^{2}\right), I^{g}\left(s, s+T_{w}, v\right)=U_{T_{w}}^{g}\left(X_{u_{i}}(s), X_{u_{i}}\left(s+T_{w}\right)\right), s=0, \tau_{i}+T_{u} .
\end{aligned}
$$

It follows from (B.13), (B.12) and the definition of $\left\{\delta_{i}\right\}_{i=1}^{\infty}$ (see (B.9)) that for each integer $i \geq 1$

$$
\begin{aligned}
& I^{g}\left(s, s+T_{w}, u_{i}\right) \leq T_{w} \mu(g)+\pi^{g}\left(X_{u_{i}}(s)\right) \\
& -\pi^{g}\left(X_{u_{i}}\left(s+T_{w}\right)\right)+2^{-i}, s=0, \tau_{i}+T_{w} .
\end{aligned}
$$

Together with (B.13), (B.10) this implies that for each integer $i \geq 1$ (B.14)

$I^{g}\left(0, \tau_{i}+2 T_{w}, u_{i}\right) \leq\left(\tau_{i}+2 T_{w}\right) \mu(g)+\pi^{g}\left(X_{u_{i}}(0)\right)-\pi^{g}\left(X_{u_{i}}\left(\tau_{i}+2 T_{w}\right)\right)+3 \cdot 2^{-i}$

For each integer $i \geq 1$ there exists $\xi_{i}^{3} \in\left[T_{w}, 2 T_{w}\right]$ such that

$$
T_{w}^{-1}\left[\xi_{i}^{2}+\xi_{i}^{3}-\xi_{i+1}^{1}\right] \text { is an integer. }
$$

We define sequences of numbers $\left\{b_{i}\right\}_{i=1}^{\infty},\left\{c_{i}\right\}_{i=1}^{\infty}$ as follows

$$
b_{1}=0, c_{i}=b_{i}+\tau_{i}+2 T_{w}, b_{i+1}=c_{i}+\xi_{i}^{3}, i=1,2, \ldots
$$


It is easy to verify that there exists $u \in W_{l o c}^{2,1}(0, \infty)$ such that for each integer $i \geq 1$

(B.17)

$$
u\left(b_{i}+t\right)=u_{i}(t), t \in\left[0, \tau_{i}+2 T_{w}\right], u\left(c_{i}+\iota\right)=w\left(\xi_{i}^{2}+\iota\right), \iota \in\left[0, \xi_{i}^{3}\right] .
$$

For each integer $i \geq 1$ we set

$$
s_{i}^{0}=b_{i}+T_{w}+s_{i} .
$$

It follows from (B.16), (B.17), (B.13), (B.11) that for each integer $i \geq 1$, for each $\xi \in\left[0, T_{w}\right)$

$$
\sup \left\{\left|X_{u}\left(s_{i}^{0}+t\right)-X_{w}(\xi+t)\right|: t \in\left[0, T_{w}\right]\right\}>\epsilon .
$$

(B.17), (B.14), (B.16) imply that $u$ is a (g)-good function. By Lemma B.1 there exists a number $T_{*}>0$ such that for each $T \geq T_{*}$ there is $\xi \in\left[0, T_{w}\right)$ such that

$$
\left|X_{u}(T+t)-X_{w}(\xi+t)\right| \leq 2^{-1} \epsilon, \quad t \in\left[0, T_{w}\right] .
$$

This is contradictory to (B.18) which holds for each integer $i \geq 1$ and each $\xi \in\left[0, T_{w}\right)$. The obtained contradiction proves the lemma.

Analogously to Lemma 3.7 in [17] we can establish the following result.

LEMmA B.3. - Let $f \in \mathfrak{M}, w \in W_{l o c}^{2,1}\left(R^{1}\right), T>0, w(t+T)=w(t)$, $t \in R^{1}, I^{f}(0, T, w)=T \mu(f), \epsilon>0$. Then there exists an integer $q \geq 1$ such that for any $\xi \in[0, T)$ there is a function $v \in W^{2,1}(0, q T)$ such that $X_{v}(0)=X_{w}(0), X_{v}(q T)=X_{w}(\xi), I^{f}(0, q T, v) \leq q T \mu(f)+\pi^{f}\left(X_{w}(0)\right)-$ $\pi^{f}\left(X_{w}(\xi)\right)+\epsilon$.

Lemma B.3 implies the following result.

Lemma B.4. - Let $\epsilon>0$. Then there exists a number $q(\epsilon)>0$ such that for each $\tau \geq q(\epsilon)$, each $\xi_{1}, \xi_{2} \in\left[0, T_{w}\right)$ there exists $v \in W^{2,1}(0, \tau)$ which satisfies $X_{v}(0)=X_{w}\left(\xi_{1}\right), X_{v}(\tau)=X_{w}\left(\xi_{2}\right)$,

$$
I^{g}(0, \tau, v) \leq \tau \mu(g)+\pi^{g}\left(X_{v}(0)\right)-\pi^{g}\left(X_{v}(\tau)\right)+\epsilon .
$$

Lemma B.4, Proposition 2.1 and the continuity of $\pi^{g}$ and $U_{T}^{g}$ imply the following extcnsion of Lemma B.3.

LemMA B.5. -Let $\epsilon>0$. Then there exist numbers $\delta, q(\epsilon)>0$ such that for each $\tau \geq q(\epsilon)$, each $x, y \in R^{2}$ satisfying

$$
d\left(x,\left\{X_{w}(t): t \in R^{1}\right\}\right) \leq \delta, d\left(y,\left\{X_{w}(t): t \in R^{1}\right\}\right) \leq \delta
$$


there exists $v \in W^{2,1}(0, \tau)$ which satisfies

$X_{v}(0)=x, X_{v}(\tau)=y, I^{g}(0, \tau, v) \leq \tau \mu(g)+\pi^{g}\left(X_{v}(0)\right)-\pi^{g}\left(X_{v}(\tau)\right)+\epsilon$.

Corollary B.1. - Let $\epsilon>0$ and let $\delta, q(\epsilon)>0$ be as guaranteed in Lemma B.5. Then for each $\tau \geq q(\epsilon)$, each $x, y \in R^{2}$ satisfying (B.19) the following relation holds

$$
U_{\tau}^{g}(x, y) \leq \tau \mu(g)+\pi^{g}\left(X_{v}(0)\right)-\pi^{g}\left(X_{v}(\tau)\right)+\epsilon .
$$

Corollary B.1 and Lemma B.2 imply the following result.

LEMma B.6. - Let $\epsilon>0$. Then there exist $\delta>0, Q>T_{w}$ such that for each $\tau \geq Q$, each $v \in W^{2,1}(0, \tau)$ which satisfies $d\left(X_{v}(s),\left\{X_{w}(t): t \in R^{1}\right\}\right) \leq$ $\delta, s=0, \tau, I^{g}(0, \tau, v) \leq U_{\tau}^{g}\left(X_{v}(0), X_{v}(\tau)\right)+\delta$ and each $s \in\left[0, \tau-T_{w}\right]$ there is $\xi \in\left[0, T_{w}\right)$ for which

$$
\left|X_{v}(s+t)-X_{w}(\xi+t)\right| \leq \epsilon, \quad t \in\left[0, T_{w}\right] .
$$

Lemmas B.6 and 2.6 imply Lemma B.

\section{REFERENCES}

[1] R.A. Adams, Sobolev spaces, Academic Press, New York, 1975.

[2] J.P. Aubin and I. Ekeland, Applied nonlinear analysis, Wiley-Interscience, New York, 1984.

[3] L.D. Berkovitz, Lower semicontinuity of integral functionals, Trans. Amer. Math. Soc., Vol. 192, 1974, pp. 51-57.

[4] D.A. CARLSON, The existence of catching-up optimal solutions for a class of infinite horizon optimal control problems with time delay, SIAM Journal on Control and Optimization, Vol. 28,1990 , pp. 402-422.

[5] D.A. CARLSON, A. Jabrane and A. Haurie, Existence of overtaking solutions to infinite dimensional control problems on unbounded time intervals, SIAM Journal on Control and Optimization, Vol. 25, 1987 , pp. 1517-1541.

[6] D.A. Carlson, A. Haurie and A. Leizarowitz, Infinite horizon optimal control, SpringerVerlag, Berlin, 1991.

[7] B.D. Coleman, M. Marcus and V.J. Mizel, On the thermodynamics of periodic phases, Arch. Rational Mech. Anal., Vol. 117, 1992, pp. 321-347.

[8] J.L. Kelley, General topology, Van Nostrand, Princeton, NJ, 1955.

[9] A. LeIZARowitz, Infinite horizon autonomous systems with unbounded cost, Appl. Math. Optim., Vol. 13, 1985 , pp. 19-43.

[10] A. LeIzARowitz and V.J. Mizel, One dimensional infinite horizon variational problems arising in continuum mechanics, Arch. Rational Mech. Anal., Vol. 106, 1989, pp. 161194.

[11] V.L. Makarov and A.M. Rubinov, Mathematical theory of econimic dynamics and equilibria, Springer -Verlag, New York, 1977.

[12] M. MARCus, Uniform estimates for a variational problem with small parameters, Arch. Rational Mech. Anal., Vol. 124, 1993 , pp. 67-98. 
[13] M. Marcus, Universal properties of stable states of a free energy model with small parameters, Cal. Var. , to appear.

[14] V.J. Mizel, L.A. Peletier and W.C. Troy, Periodic phases in second order materials, Preprint, 1997.

[15] A.J. ZaSLAVSKI, The existence of periodic minimal energy configurations for onedimensional infinite horizon variational problems arising, in continuum mechanics. J. Math. Anal. Appl., Vol. 194, 1995, pp. 459-476.

[16] A.J. ZASLAVSKI, The existence and structure of extremals for a class of second order infinite horizon variational problems, J. Math. Anal. Appl., Vol. 194, 1995, pp. 660-696.

[17] A.J. ZASLAVSKI, Structure of extremals for one-dimensional variational problems arising in continuum mechanics, J. Math. Anal. Appl., Vol. 198, 1996, pp. 893-921.

[18] A.J. ZASLAVSKI, The asymptotic turnpike property and the uniqueness of a periodic minimal solution for one-dimensional variational problems, Preprint, 1996.

(Manuscript received February 28, 1997; Revised version received November 20,1997.) 